\title{
Problemi relativi alle caratteristiche per sistemi di equazioni semilineari a derivate parziali (*).
}

\author{
Maria Cinquini Otbrario (Pavia)
}

Sunto. - L considerato il sistema di tipo iperbolico di equazioni semilineari a derivate parziali

$$
\sum_{j=1}^{m} A_{i j}(x, y)\left(p_{j}(x, y)+\varrho_{i}(x, y) q_{j}(x, y)\right)=f_{i}\left(x, y ; z_{1}(x, y), \ldots, z_{m}(x, y)\right), \quad(i=1, \ldots, m)
$$

nella teoria delle ourve caratteristiche del sistema (I), imtese in senso generalizzato, sono dimostrati un teorema di unicità e un teorema di dipendenza continua dai dati, considerando come soluzione (in senso generalizzato) del sistema (I) una m-pla di funzioni $z_{1}(x, y), \ldots, z_{m}(x, y)$ lipschitziane nel complesso delle variabili e saddisfacenti il sistema (I) quasi ovunque mel proprio campo di definizione.

\section{Introduzione.}

Molti anni fa mi ero occupata, in successivi lavori, della teoria delle caratteristiche per un sistema di tipo iperbolico di equazioni a derivate parziali in due variabili indipendenti, sia nel caso in cui le equazioni stesse siano quasi lineari, sia nel caso in cui esse siano non lineari; la teoria era sviluppata, partendo da alcuni teoremi di esistenza e di unicità, ottenuti nell'indirizzo classico $\left(^{(1)}\right.$.

Successive ricerche, sia mie che di S. CINQUINI ( $\left.{ }^{2}\right)$, sono state rivolte ad estendere alle equazioni a derivate parziali l'ordine di idee che C. Carathéodory $\left({ }^{3}\right)$ aveva introdotto per le equazioni differenziali ordinarie; in particolare ho ottenuto, sotto ipotesi molto ampie, teoremi di esistenza e di unicità relativi al problema di

(*) Entrato in Redazione il 18 giugno 1975.

(1) Una breve esposizione dei risultati di tali ricerche è contenuta nel volume M. CINQUINI Cibrario, S. Crnquini [1] (i numeri tra [ ] si riferiscono alla bibliografia posta alla fine della presente memoria), Cap. V, $\$ 4$, nn. 13-17, pp. 430-44I; $\$ 5$, n. 20, pp. 444-449. Per le indicazioni bibliografiche dei lavori di M. CINQUINI CIBRARIo sull'argomento rinviamo per brevità ai l.c. di tale volume.

(2) Una esposizione di alcune di tali ricerche è contenuta nel volume citato in (1), Cap. IV, pp. 301-384, e anche, nel caso di due variabili indipendenti, Cap. V, § 2, n. 6, pp. 397-421. Cfr. anche M. Cinquini Cibrario [3]-[9], S. Cinquint [1]-[8]; si fa presente al lettore che nella bibliografia posta alla fine della presente Memoria sono citati soltanto i lavori di tali autori, posteriori al volume citato in $\left(^{1}\right)$; per $i$ lavori anteriori si rimanda per brevità alla bibliografia posta alla fine dei Cap. IV e $V$ di tale volume.

(3) C. Caratheodory [1], Cap. XI, pp. 665-688. 
CAUCHY per il sistema di equazioni quasi lineari in $r+1$ variabili indipendenti

$$
\begin{aligned}
\sum_{j=1}^{m} A_{i j}\left(x, y_{1}, \ldots, y_{r} ;\right. & \left.z_{1}(\ldots), \ldots, z_{m i}(\ldots)\right)\left(\frac{\partial z_{j}\left(x, y_{1}, \ldots, y_{r}\right)}{\partial x}+\right. \\
& \left.+\sum_{k=1}^{r} \varrho_{i k}(\ldots) \frac{\partial z_{j}\left(x, y_{1}, \ldots, y_{r}\right)}{\partial y_{k}}\right)=f_{i}(\ldots . .), \quad(i=1, \ldots, m)
\end{aligned}
$$

nel campo funzionale costituito dalle $m$-ple di funzioni $z_{1}\left(x, y_{1}, \ldots, y_{r}\right), \ldots, z_{m}\left(x, y_{1}\right.$, $\left.\ldots, y_{r}\right)$, assolutamente continue in $x$, lipschitziane nel complesso delle variabili $\left(y_{1}, \ldots, y_{r}\right)$ e soddisfacenti il sistema stesso quasi ovunque nel proprio campo di definizione; ho pure esteso i risultati a un sistema non lineare del tipo

$$
\frac{\partial z_{i}\left(x, y_{1}, \ldots, y_{r}\right)}{\partial x}=f_{i}\left(x, y_{1}, \ldots, y_{r} ; z_{1}(\ldots), \ldots, z_{m}(\ldots)\right), \quad(i=1, \ldots, m)
$$

D'altra parte S. Cinguin ha dimostrato teoremi di unicità relativi al problema di CAUCHY per i sistemi $(a)$ e $(b)$ sotto ipotesi e in campi funzionali più ampi di quelli considerati da me.

Riprendendo i miei lavori citati in (1), relativi alla teoria delle caratteristiche nel caso di due variabili indipendenti, ho pensato di sviluppare tale teoria nell'ordine di idee dei lavori citati in $\left(^{2}\right)$; nel presente lavoro sono stabiliti un teorema di unicità e un teorema di dipendenza continua dai dati per il sistema di tipo iperbolico di equazioni semilineari a derivate parziali in due variabili indipendenti, già ridotto $\iota_{n}$ forma caratteristica

dove

$$
\begin{aligned}
\sum_{j=1}^{m} A_{i j}(x, y)\left(p_{j}(x, y)+\varrho_{i}(x, y)\right. & \left.q_{j}(x, y)\right)= \\
& =f_{i}\left(x, y ; z_{1}(x, y), \ldots, z_{m}(x, y)\right), \quad(i=1, \ldots, m),
\end{aligned}
$$

$$
p_{j}(x, y)=\frac{\partial z_{j}(x, y)}{\partial x}, \quad q_{j}(x, y)=\frac{\partial z_{j}(x, y)}{\partial y}, \quad(i=1, \ldots, m) .
$$

La natura dei problemi, studiati nel presente lavoro, ha richiesto ipotesi alquanto più restrittive, rispetto a quelle introdotte nelle ricerche, citate in ${ }^{2}{ }^{2}$. Nel $\S 1$, dopo alcune considerazioni introduttive, è dimostrato un teorema di unicità, nel $\S 2$ un teorema di dipendenza continua dai dati.

\section{§ 1.}

\section{1. - Preliminari.}

a) Nel presente lavoro è intesa come soluzione (in senso generalizzato) del sistema semilineare $(I)$ in un campo $\Delta$ del piano $(x, y)$ una $m$-pla di funzioni

$$
z_{1}(x, y), z_{2}(x, y), \ldots, z_{m}(x, y), \quad((x, y) \text { in } \Delta)
$$


le quali sono lipschitziane nel complesso delle variabili e soddisfano il sistema (I) quasi ovunque nel campo $\Delta\left(^{4}\right)$.

b) Le funzioni

$$
\varrho_{1}(x, y), \varrho_{2}(x, y), \ldots, \varrho_{m}(x, y)
$$

sono, come è noto, le radici caratteristiche del sistema (I).

Le fanzioni (2) siano continue nel loro campo di definizione, e inoltre valga una delle seguenti ipotesi

1) Le $m$ radici caratteristiche (2) siano a due a due distinte in ogni punto del loro campo di definizione.

2) Se le radici caratteristiche (2) non sono tutte distinte, l'ordine di molteplieità di ognuna di esse sia costante in tutto il loro campo di definizione. Indicate con

$$
\sigma_{1}(x, y), \sigma_{2}(x, y), \ldots, \sigma_{\mu}(x, y)
$$

le radici caratteristiche del sistema $(\mathrm{I})$ a due a due distinte $e$ con $v_{1}, v_{2}, \ldots, v_{\mu}$ i loro ordini di molteplicità $\left(v_{1}+v_{2}+\ldots+v_{\mu}=m\right)$, valgano le

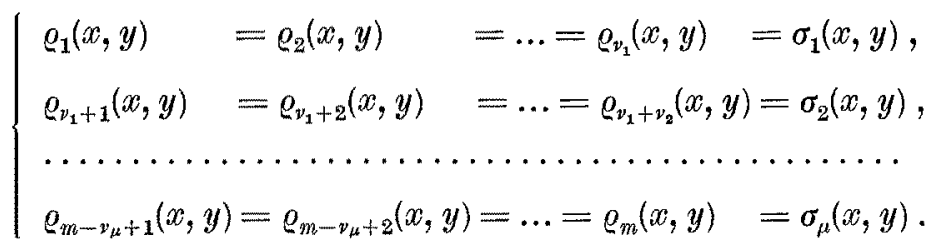

Nel seguito, anche nel caso delle radici caratteristiche multiple, il sistema semilineare è scritto nella forma (I), tenendo presenti le posizioni (4).

c) Una $(m+1)$-pla di funzioni

$$
y=\eta_{1}(x), \quad z_{i}=Z_{i}(x), \quad(i=1, \ldots, m),
$$

definite in un intervallo $\left(x^{\prime}, x^{\prime \prime}\right)$, con $\eta_{1}(x)$ di classe $C^{(1)}$ e $Z_{i}(x)$ lipschitziane in tale intervallo, è detta eurva caratteristica (in senso generalizzato) del sistema (I) corrispondente alla radice caratteristica $\sigma_{1}(x, y)$ di molteplicità $\nu_{1}$, se per ogni $x$ di $\left(x^{\prime}, x^{\prime \prime}\right)$ tutti i punti $\left(x, \eta_{1}(x), Z_{1}(x), \ldots, Z_{m}(x)\right)$ appartengono al campo di definizione delle funzioni $A_{i j}(x, y), \varrho_{i}(x, y), f_{i}\left(x, y ; z_{1}, \ldots, z_{m}\right)$ e inoltre la

$$
\eta_{1}^{\prime}(x)=\sigma_{1}\left(x, \eta_{1}(x)\right)
$$

(4) Il campo funzionale considerato è quindi meno ampio di quello introdotto nei lavori citati in (2) relativi al problema di Cauchy; ciò è nella natura dei problemi relativi alla teoria delle caratteristiche, nella quale le variabili $(x, y)$ hanno lo stesso ufficio. 
è soddisfatta per ogni $x$ di $\left(x^{\prime}, x^{\prime \prime}\right)$, e le

$$
\sum_{j=1}^{m} A_{i j}\left(x, \eta_{1}(x)\right) Z_{j}^{\prime}(x)=f_{i}\left(x, \eta_{1}(x) ; Z_{1}(x), \ldots, Z_{m}(x)\right), \quad\left(i=1, \ldots, v_{1}\right)
$$

sono soddisfatte per quasi tutti gli $x$ di $\left(x^{\prime}, x^{\prime \prime}\right)\left(^{5}\right)$.

d) Si dice che la curva caratteristica (5) appartiene all'integrale (1) del sistema (I), se esiste un intervallo $\left(x_{1}, x_{2}\right)$, con $\left.x^{\prime} \leqslant x_{1}<x_{2} \leqslant x^{\prime \prime}\right)$, tale che l'arco di curva $y=\eta_{1}(x),\left(x_{1}, x_{2}\right)$ appartiene al campo $\Delta$ e inoltre valgono le

$$
z_{i}\left(x, \eta_{1}(x)\right)=Z_{i}(x), \quad(i=1, \ldots, m)
$$

\section{2. - Teorema di unicità.}

Le funzioni $A_{i j}(x, y),(i, j=1, \ldots, m)$ siano definite nel campo

$$
D_{\infty}:-a_{1} \leqslant x \leqslant a_{2}, \quad-\infty<y+\infty, \quad\left(a_{1}>0, a_{2}>0\right)
$$

e siano ivi lipschitziane nel complesso delle variabili. Se $A$ è il determinante, $i$ aui elementi sono $A_{i j}(x, y),(i, j=1, \ldots, m)$, in tutto $D_{\infty}$ sia

$$
A=1 \text {. }
$$

Le funzioni $\varrho_{i}(x, y),(i=1, \ldots, m)$ siano definite nel campo $D_{\infty}$ e siano ivi continue nel complesso delle variabili; in ogni punto del campo $D_{\infty}$ esistano finite le derivate $\partial \varrho_{i}(x, y) / \partial y,(i=1, \ldots, m)$, le quali, in corrispondenza ad ogni y reale sono quasi continue in $x$ in $\left(-a_{1}, a_{2}\right), e$, in corrispondenza ad ogni $x$ di $\left(-a_{1}, a_{2}\right)$, sono continue in $y$; esistano due funsioni $M(x), L(x)$, quasi continue, non negative e integrabili $\left(^{6}\right)$, tali che, in corrispondenza a quasi tutti gli $x d i\left(-a_{1}, a_{2}\right)$, sia

$$
\left|\varrho_{i}(x, y)\right| \leqslant M(x), \quad(i=1, \ldots, m)
$$

per tutti gli y reali, $e$

$$
\left|\varrho_{i}(x, y)-\varrho_{i}(x, \bar{y})\right| \leqslant L(x)|y-\bar{y}|, \quad(i=1, \ldots, m)
$$

per tutte le coppie $(y, \bar{y})$ di numeri reali, così che, per quasi tutti gli $x$ di $\left(-a_{1}, a_{2}\right)$, ̀̀

$$
\left|\frac{\partial \varrho_{i}(x, y)}{\partial y}\right| \leqslant L(x), \quad(i=1, \ldots, m) .
$$

(5) Nella teoria sviluppata nei nostri lavori indicati in $\left(^{(1)}\right.$ le $Z_{i}(x)$ sono supposte di classe $C^{(1)}$ in $\left(x^{\prime}, x^{\prime \prime}\right)$, e le $\left(6_{2}\right)$ sono supposte soddisfatte in ogni punto dell'intervallo $\left(x^{\prime}, x^{\prime \prime}\right)$.

${ }^{6}{ }^{6}$ L'integrabilità è intesa nel senso di Lebesgue. 
Le funzioni

$$
f_{i}\left(x, y ; z_{1}, \ldots, z_{m}\right), \quad(i=1, \ldots, m)
$$

siano definite nel campo

$$
C_{\infty}:-a_{1} \leqslant x \leqslant a_{2},-\infty<y<+\infty,-\infty<z_{1}<+\infty, \ldots,-\infty<z_{m}<+\infty
$$

e siano ivi continue nel complesso delle variabili; esista una costante $L_{1}$ tale che, in corrispondenza ad ogni punto $(x, y)$ del campo $D_{\infty}$, valgano le

$$
\left|f_{i}\left(x, y ; z_{1}, \ldots, z_{m}\right)-f_{i}\left(x, y ; \bar{z}_{1}, \ldots, \bar{z}_{m}\right)\right| \leqslant L_{1} \sum_{j=1}^{m}\left|z_{j}-\bar{z}_{j}\right|,(i=1, \ldots, m)
$$

per tutte le coppie di m-ple reali $\left(z_{1}, \ldots, z_{m}\right),\left(\bar{z}_{1}, \ldots, \bar{z}_{m}\right)$.

Valga l'ipotesi 1) o più in generale 2) del $n .1$ b); sia $v_{1}$ l'ordine di molteplicità della radice caratteristica $\sigma_{1}(x, y)$, cosi che valgano le prime $v_{1}$ tra le $(4)$, e per $-a_{1} \leqslant$ $\leqslant x \leqslant a_{2}$ sia

$$
y=\eta_{1}(x), \quad z_{i}=Z_{i}(x), \quad(i=1, \ldots, m)
$$

una curva caratteristica del sistema corrispondente a tale radice con $\eta_{1}(x)$ di classe $C^{(1)}$ e $Z_{i}(x)$ lipschitziane in $\left(-a_{1}, a_{2}\right)$.

La funzione di classe $C^{(1)}$ in $\left(-a_{1}, a_{2}\right)$

$$
y=\eta_{2}(x), \quad\left(-a_{1} \leqslant x \leqslant a_{2}\right)
$$

soddisfi in tutto $\left(-a_{1}, a_{2}\right)$ l'equazione differenziale ordinaria

$$
\eta_{2}^{\prime}(x)=\sigma_{2}\left(x, \eta_{2}(x)\right)
$$

Valga inoltre la $\left(^{7}\right)$

$$
\eta_{1}(0)=\eta_{2}(0)=0
$$

Siano assegnate in $\left(-a_{1}, a_{2}\right)$ le funzioni continue $b_{i j}(x),\left(i=1, \ldots, \nu_{1} ; j=1, \ldots, m\right)$, $G_{i}(x),\left(i=1, \ldots, v_{1}\right)$, soddisfacenti in tutto $\left(-a_{1}, a_{2}\right)$ la

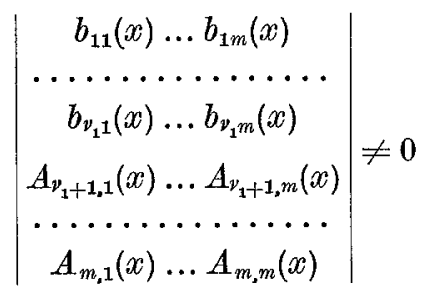

( $\left.{ }^{7}\right)$ La condizione (16) evidentemente non è restrittiva; è imposto che le curve $y=\eta_{1}(x)$, $y=\eta_{2}(x)$ si incrocino in un punto interno all'intervallo $\left(-a_{1}, a_{2}\right)$. 
e inoltre le

$$
\sum_{j=1}^{m} b_{i j}(0) Z_{j}(0)=G_{i}(0), \quad\left(i=1, \ldots, v_{1}\right)
$$

In tali ipotesi non può esistere più di una m-pla di funzioni

$$
z_{1}=z_{1}(x, y), z_{2}=z_{2}(x, y), \ldots, z_{m}=z_{m}(x, y)
$$

le quali sono definite in un opportuno eampo $\Delta\left({ }^{8}\right)$, sono ivi lipschitziane, soddisfano in quasi tutto $\Delta$ il sistema

$$
\begin{aligned}
\sum_{j=1}^{m} A_{i j}(x, y)\left(p_{j}(x, y)+\varrho_{i}(x, y) q_{j}(x, y)\right) & = \\
& =f_{i}\left(x, y ; z_{1}(x, y), \ldots, z_{m}(x, y)\right), \quad(i=1, \ldots, m),
\end{aligned}
$$

e inoltre soddisfano le

$$
z_{i}\left(x, \eta_{1}(x)\right)=Z_{i}(x), \quad(i=1, \ldots, m)
$$

identicamente in $\left(-a_{1}, a_{2}\right)$, e nei punti della curva (14) appartenenti al campo $\Delta$ soddisfano le

$$
\sum_{j=1}^{\text {mix }} b_{i j}(x) z_{j}\left(x, \eta_{2}(x)\right)=G_{i}(x), \quad\left(i=1, \ldots, v_{1}\right),
$$

oioè non può esistere più di un integrale del sistema (I) (in senso generalizzato), a cui appartiene la ourva caratteristica (5), e che soddisfa le $v_{1}$ condizioni (20) nei punti della ourva (14), appartenenti al campo $\Delta$.

a) Poichè $\sigma_{1}(x, y), \sigma_{2}(x, y)$ sono distinte in tutto il campo $D_{\infty}$, si supponga, per fissare le idee, che sia

$$
\sigma_{1}(x, y)<\sigma_{2}(x, y)
$$

in tutto $D_{\infty}$; allora, tenuto conto della (16), le curve

$$
\gamma_{1}: y=\eta_{1}(x),\left(-a_{1} \leqslant x \leqslant a_{2}\right) ; \quad \gamma_{2}: y=\eta_{2}(x),\left(-a_{1} \leqslant x \leqslant a_{2}\right)
$$

si incrociano nell'origine e non possono avere altri punti in comune $\left({ }^{9}\right)$; è dunque

$$
\eta_{1}(x)>\eta_{2}(x),\left(-a_{1} \leqslant x<0\right) ; \quad \eta_{1}(x)<\eta_{2}(x),\left(0<x \leqslant a_{2}\right) .
$$

(8) Il campo $\Delta$ viene definito nel corso della dimostrazione (cfr. capoverso $d$ )).

(9) Cfr. M. Cinquinr Cubrario [1], § 1, Teorema III, pp. 27-28, e [2], Teorema I, pp. 115-116. 
b) Indicate (qui e spesso nel seguito) con $(X, Y)$ le coordinate correnti, sia $\sigma_{r}(X, Y)(1 \leqslant r \leqslant \mu)$ una qualsiasi delle radici caratteristiche del sistema $(I)$; in virtù dei noti teoremi di esistenza e di unicità di C. CARATHÉodory $\left({ }^{10}\right)$, se $(x, y)$ è un punto fissato del campo $D_{\infty}$, l'equazione differenziale, scritta in forma integrale

$$
g_{r}(X ; x, y)=y+\int_{x}^{x} \sigma_{r}\left(t, g_{r}(t ; x, y)\right) d t
$$

ammette un'unica soluzione, definita nell'intervallo $-a_{1} \leqslant X \leqslant a_{2}$; in particolare, in virtù delle $\left(6_{1}\right),(15),(16)\left({ }^{11}\right)$, risulta

$$
\eta_{1}(X)=g_{1}(X ; 0,0), \quad \eta_{2}(X)=g_{2}(X ; 0,0), \quad\left(-a_{1} \leqslant X \leqslant a_{2}\right) .
$$

Inoltre se è $1 \leqslant s \leqslant \mu, s \neq r$, le curve

$$
Y=g_{r}(X ; x, y), \quad Y=g_{s}(X ; x, y), \quad\left(-a_{1} \leqslant X \leqslant a_{2}\right)
$$

non hanno punti in comune nel campo

$$
-a_{1} \leqslant X \leqslant a_{2}, \quad-\infty<Y<+\infty
$$

all'infuori del punto $(x, y)\left({ }^{12}\right)$. In tutto il campo

$$
-a_{1} \leqslant X \leqslant a_{2}, \quad-a_{1} \leqslant x \leqslant a_{2}, \quad-\infty<y<+\infty
$$

esistono finite le derivate

$$
\begin{aligned}
& \frac{\partial g_{r}(X ; x, y)}{\partial x}=-\sigma_{r}(x, y) \exp \left[I_{r}(X ; x, y)\right] \\
& \frac{\partial g_{r}(X ; x, y)}{\partial y}=\exp \left[I_{r}(X ; x, y)\right]
\end{aligned}
$$

dove

$$
I_{r}(X ; x, y)=\int_{x}^{X} \frac{\partial \sigma_{r}\left(t, g_{r}(t ; x, y)\right)}{\partial Y} d t,
$$

e tali derivate sono continue nel complesso delle variabili $(X ; x, y)\left({ }^{13}\right)$.

(10) C. Carathéodory [1], Cap. XI, pp. 665-688; in particolare n. 582, pp. 672-674, n. 583, pp. 674-675; poichè le $\sigma_{r}(X, Y)$ sono supposte continue nel complesso delle variabili, $g_{r}(X ; x, y)$ $\grave{e ̀ ~ s o l u z i o n e ~ d e l l a ~ e q u a z i o n e ~ d i f f e r e n z i a l e ~ i n ~ s e n s o ~ c l a s s i c o . ~}$

(11) Nelle $\left(6_{1}\right)$, (15) va assunta $X$ come coordinata corrente; la $\left(6_{1}\right)$ è soddisfatta per $-a_{1} \leqslant X \leqslant a_{2}$.

(12) Cfr. 1.c. in $\left(^{9}\right)$.

(19) C. CaRathéodory [1], Cap. XI, nn. 589, 590, 591, pp. 682-687; M. Volpato [1], e anche E. J. Mc SHaxe [1], Cap. V, n. 39, pp. 216-217; Cap. IX, n. 69, pp. 348-365. I risultati citati devono essere completati con considerazioni, che tengono conto delle ipotesi fatte sulle $\sigma_{r}(x, y)$. 
c) Tenute presenti le (24), si considerino le curve $\sigma_{1}, \sigma_{2}$ di equazioni rispettive

$$
\begin{aligned}
& Y=g_{1}\left(X ;-a_{1}, \eta_{2}\left(-a_{1}\right)\right), \quad\left(-a_{1} \leqslant X \leqslant a_{2}\right), \\
& Y=g_{1}\left(X ; a_{2}, \eta_{2}\left(a_{2}\right)\right), \quad\left(-a_{1} \leqslant X \leqslant a_{2}\right),
\end{aligned}
$$

uscenti dagli estremi della curva $\gamma_{2}$, e le curve $\sigma_{3}, \sigma_{4}$ di equazioni

$$
\begin{aligned}
& Y=g_{2}\left(X ;-a_{1}, \eta_{1}\left(-a_{1}\right)\right), \quad\left(-a_{1} \leqslant X \leqslant a_{2}\right), \\
& Y=g_{2}\left(X ; a_{2}, \eta_{1}\left(a_{2}\right)\right), \quad\left(-a_{1} \leqslant X \leqslant a_{2}\right),
\end{aligned}
$$

uscenti dagli estremi della curva $\gamma_{1}$.

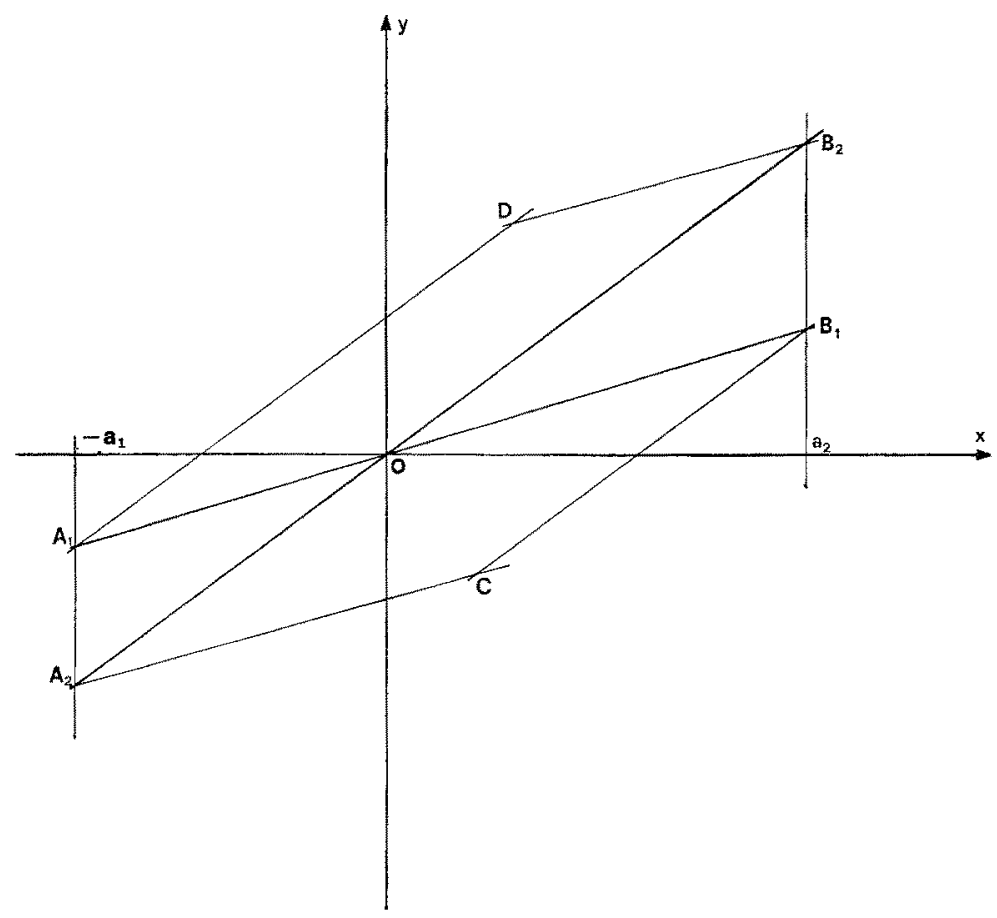

Figura 1

Con semplici considerazioni si prova che le curve $\left(27_{1}\right)$ e $\left(27_{4}\right)$ hanno un punto in comune di ascissa $X_{1}$, con $a_{1}<X_{1}<a_{2}$, e ordinata $Y_{1}$ con $Y_{1}<\eta_{1}\left(X_{1}\right), Y_{1}<\eta_{2}\left(X_{1}\right)$, e analogamente che le curve $\left(27_{2}\right)$ e $\left(27_{3}\right)$ hanno un punto in comune di ascissa $X_{2}$, $\left(-a_{1}<X_{2}<a_{2}\right)$ e ordinata $Y_{2}$ con $Y_{2}>\eta_{1}\left(X_{2}\right), Y_{2}>\eta_{1}\left(X_{2}\right)$. Indicate sempre con $(X, Y)$ le variabili, sia $A^{[11}$ il campo chiuso (cfr. fig. 1), limitato da archi delle curve (27) e dai segmenti

$$
X=-a_{1}, \quad \eta_{2}\left(-a_{1}\right) \leqslant Y \leqslant \eta_{1}\left(-a_{1}\right) ; \quad X=a_{2}, \quad \eta_{1}\left(a_{2}\right) \leqslant Y \leqslant \eta_{2}\left(a_{2}\right) .
$$


Se $(x, y)$ è un punto del campo $4^{\mathrm{rn}}$, la curva

$$
Y=g_{2}(X ; x, y), \quad\left(-a_{1} \leqslant X \leqslant a_{2}\right)
$$

incontra la curva $\gamma_{1}$ in un unico punto di ascissa $X=\psi(x, y)$, e la curva

$$
Y=g_{1}(X ; x, y), \quad\left(-a_{1} \leqslant X \leqslant a_{2}\right)
$$

incontra la curva $\gamma_{2}$ in un unico punto di ascissa $X=\chi(x, y)\left({ }^{14}\right)$.

Tenuto conto delle $(24)$, le funzioni $\psi(x, y), \chi(x, y)$ soddisfano le

$$
\begin{aligned}
& g_{2}(\psi(x, y) ; x, y)=\eta_{1}(\psi(x, y)), \\
& g_{1}(\chi(x, y) ; x, y)=\eta_{2}(\chi(x, y)) .
\end{aligned}
$$

Le ipotesi fatte circa le funzioni $\sigma_{1}(x, y), \sigma_{2}(x, y)$ assicurano che le funzioni $\psi(x, y), \chi(x, y)$ sono continue, assieme alle loro derivate prime nel campo $4^{[1]}$; con qualche calcolo, tenuto conto delle (25), si ottengono le

$$
\left\{\begin{array}{l}
\frac{\partial \psi(x, y)}{\partial x}=\frac{-\sigma_{2}(x, y) \exp \left[I_{2}(\psi(x, y) ; x, y)\right]}{\sigma_{1}\left(\psi(x, y), \eta_{1}(\psi(x, y))\right)-\sigma_{2}\left(\psi(x, y), \eta_{1}(\psi(x, y))\right)} \\
\frac{\partial \psi(x, y)}{\partial y}=\frac{\exp \left[I_{2}(\psi(x, y) ; x, y)\right]}{\sigma_{1}\left(\psi(x, y), \eta_{1}(\psi(x, y))\right)-\sigma_{2}\left(\psi(x, y), \eta_{1}(\psi(x, y))\right)} \\
\frac{\partial \chi(x, y)}{\partial x}=\frac{-\sigma_{1}(x, y) \exp \left[I_{1}(\chi(x, y) ; x, y)\right]}{\sigma_{2}\left(\chi(x, y), \eta_{2}(\chi(x, y))\right)-\sigma_{1}\left(\chi(x, y), \eta_{2}(\chi(x, y))\right)} \\
\frac{\partial \chi(x, y)}{\partial y}=\frac{\exp \left[I_{1}(\chi(x, y) ; x, y)\right]}{\sigma_{2}\left(\chi(x, y), \eta_{2}(\chi(x, y))\right)-\sigma_{1}\left(\chi(x, y), \eta_{2}(\chi(x, y))\right)}
\end{array} .\right.
$$

d) Indicate ancora con $(X, Y)$ le coordinate correnti, distinguiamo quattro casi, tenendo presente la $(21)$ e il fatto che le $\sigma_{r}(X, Y),(r=1, \ldots, \mu)$ sono tutte distinte.

1) Sia $\sigma_{1}(X, Y)<\sigma_{2}(X, Y)<\sigma_{r}(X, Y),(r=3, \ldots, \mu)$ in tutto il campo - $a_{1} \leqslant$ $\leqslant X \leqslant a_{2},-\infty<Y<+\infty$; allora, se $(x, y)$ è un punto qualsiasi del campo $\Delta^{[1]}$, la curva $Y=g_{r}(X ; x, y)$ (dove $r$ è uno qualsiasi dei numeri $3, \ldots, \mu$ ) incontra in un punto di ascissa $X=\psi_{r}(x, y)$ la curva $\gamma_{1}$, come si può vedere immediatamente; nel presente caso il campo $\Delta$, in cui viene dimostrato il teorema di unicità, coincide con il campo $\Delta^{[1]}$, definito nel capoverso e) (cfr. fig. 1 ; il campo $\Delta \equiv \Delta^{[1]}$ è il campo ${ }^{15}$ ) $\left.A_{1} A_{2} O B_{1} B_{2} D\right)$.

(14) Ciò segue da semplici considerazioni circa l'andamento delle curve in questione e anche dai risultati citati in $\left({ }^{9}\right)$.

$\left({ }^{15}\right)$ Nelle figure, per semplicità, le curve caratteristiche sono rappresentate da rette; inoltre nei campi $\Delta^{(1)}$ e $\Delta$ sono assunte come coordinate correnti $(x, y)$. 
2) Esistono aleune tra le $\sigma_{r}(X, Y),(r=3, \ldots, \mu)$ minori di $\sigma_{1}(X, Y)$ e sia $\sigma_{h}(X, Y)$ la massima tra esse; sia inoltre $\sigma_{k}(X, Y)$ la minima tra le $\sigma_{r}(X, Y)$ maggiori di $\sigma_{1}(X, Y)$; nel presente caso 2$)$ sia $k>2$; si considerino le curve

$$
\begin{aligned}
& Y=g_{k}\left(X ;-a_{1}, \eta_{1}\left(-a_{1}\right)\right), \quad\left(-a_{1} \leqslant X \leqslant a_{2}\right), \\
& Y=g_{h}\left(X ; a_{2}, \eta_{1}\left(a_{2}\right)\right), \quad\left(-a_{1} \leqslant X \leqslant a_{2}\right),
\end{aligned}
$$

delle quali la seconda incontra certamente in un punto $M_{1}$ l'arco della curva $Y=$ $=\eta_{2}(X)$, corrispondente a $X>0$, la prima può incontrarlo in un punto $M_{2}$ oppure no; nel primo caso si indichi con $M$ quello tra i punti $M_{1}, M_{2}$ di ascissa minore, nel secondo si indichi con $M$ il punto $M_{1}$. Indicate con $\left(x_{3}, \eta_{2}\left(a_{3}\right)\right)$ le coordinate del punto $M$ e supposto, p. es., che il punto $M$ appartenga alla curva $\left(30_{2}\right)$, si consideri l'arco della curva

$$
Y=g_{1}\left(X ; a_{3}, \eta_{2}\left(a_{3}\right)\right), \quad\left(-a_{1} \leqslant X \leqslant a_{2}\right),
$$

corrispondente a $-a_{1} \leqslant X \leqslant a_{3}$, il quale incontra certamente in un punto la curva $\left(30_{1}\right)\left({ }^{16}\right)$. In modo analogo si considerino le curve

$$
\begin{array}{ll}
Y=g_{h}\left(X ;-a_{1}, \eta_{1}\left(-a_{1}\right)\right), & \left(-a_{1} \leqslant X \leqslant a_{2}\right), \\
Y=g_{h}\left(X ; a_{2}, \eta_{1}\left(a_{2}\right)\right), & \left(-a_{1} \leqslant X \leqslant a_{2}\right),
\end{array}
$$

delle quali la prima incontra in un punto $N_{1}$ l'arco della curva $Y=\eta_{2}(X)$, corrispondente a $X<0$, la seconda può incontrarlo in un punto $N_{2}$ oppure non incontrarlo; nel primo caso si indichi con $N$ quello tra i punti $N_{1}$ e $N_{2}$, che ha minore il valore assoluto dell'ascissa, nel secondo si indichi con $N$ il punto $N_{1}$; siano $\left(a_{4}, \eta_{2}\left(a_{4}\right)\right)$ le coordinate del punto $N$. Supposto, p. es., che $N$ appartenga alla curva $\left(32_{1}\right)$ si consideri l'arco della curva

$$
Y=g_{1}\left(X ; a_{4}, \eta_{2}\left(a_{4}\right)\right), \quad\left(-a_{1} \leqslant X \leqslant a_{2}\right),
$$

corrispondente ad $a_{4} \leqslant X \leqslant a_{2}$, il quale incontra in un punto $K$ la curva $\left(32_{2}\right)\left({ }^{17}\right)$. Il campo $\Delta$ è il campo, del cui contorno fanno parte gli archi considerati delle curve $\left(30_{1}\right),\left(30_{2}\right),(31),\left(32_{1}\right),\left(32_{2}\right),(33)$ (cfr. fig. 2, campo $\left.A_{1} N K B_{1} M H\right)$.

3) Valgano le ipotesi del precedente eapoverso, ma sia $k=2$; in tale caso il punto $M$ coincide con il punto $M_{1}$ e il punto $N$ con il punto $N_{1}$; considerati ancora l'arco della curva (31) corrispondente a $-a_{1} \leqslant X \leqslant a_{3}$, e l'arco della curva (33) corrispondente ad $a_{4} \leqslant X \leqslant a_{2}$, essi incontrano rispettivamente le curve $\left(27_{3}\right)$ e $\left(27_{4}\right)$ nei punti $H$ e $K$; il campo $\Delta$ è limitato da archi delle curve di equazioni $\left(27_{3}\right),\left(27_{4}\right)$, $\left(30_{2}\right),(31),\left(32_{1}\right),(33)$.

(16) Se $M$ appartiene alla curva $\left(30_{1}\right)$, si considera l'arco della curva (31) corrispondente ad $a_{3} \leqslant X \leqslant a_{2}$, il quale incontra in un punto $H$ la curva $\left(30_{2}\right)$.

$\left({ }^{17}\right)$ Se $N$ appartiene alla curva $\left(32_{2}\right)$, si considera l'arco della curva (33), corrispondente $a-a_{1} \leqslant X \leqslant a_{4}$, il quale incontra in un punto $K$ la curva $\left(32_{1}\right)$. 


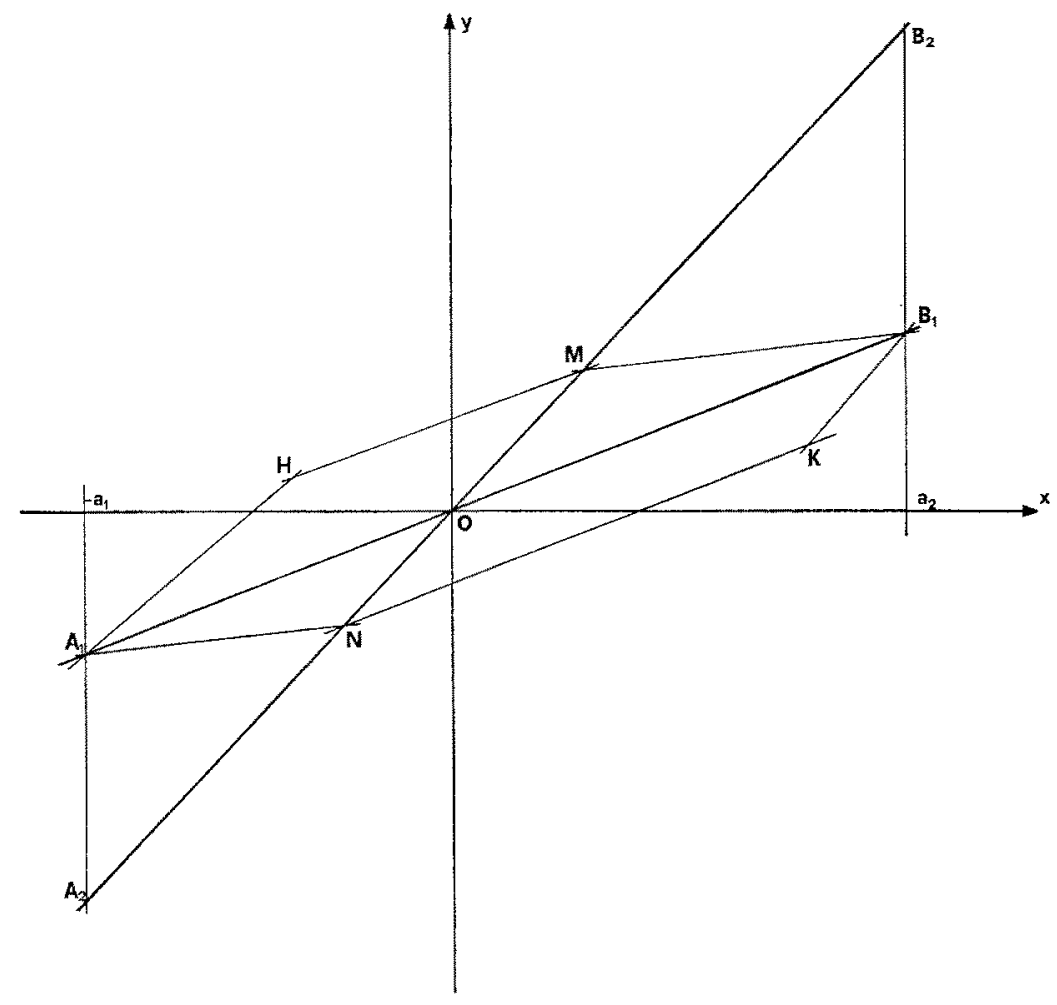

Figura 2

4) Sia $\sigma_{1}(X, Y)<\sigma_{r}(X, Y),(r=2, \ldots, \mu)$; sia $\sigma_{k}(X, Y)$ la minima tra le $\sigma_{r}(X, Y),(r=2, \ldots, \mu)$, e sia inoltre $k>2\left({ }^{18}\right)$. Se la curva di equazione $\left(30_{1}\right)$ incontra in un punto $M\left(a_{3}, \eta_{2}\left(a_{3}\right)\right)$ l'arco della curva $Y=\eta_{2}(X)$ corrispondente a $0<X \leqslant a_{2}$, si considerano l'arco della curva (31) corrispondente ad $a_{3} \leqslant X \leqslant a_{2}$ e il punto $K$ di coordinate $\left(a_{2}, g_{1}\left(a_{2} ; a_{3}, \eta_{2}\left(a_{3}\right)\right)\right)$; se la curva $\left(30_{1}\right)$ non incontra la curva $Y=\eta_{2}(X)$, incontra certamente in un punto $K^{[1]}$ la curva di equazione $\left(27_{2}\right)$. Analogamente se la.curva di equazione $\left(32_{2}\right)$ incontra in un punto $N\left(a_{4}, \eta_{2}\left(a_{4}\right)\right)$ l'arco della curva $Y=\eta_{2}(X)$ corrispondente a $-a_{1} \leqslant X<0$, si considerano l'arco della curva di equazione (33) corrispondente a $-a_{1} \leqslant X \leqslant a_{4}$ e il punto $H$ di coordinate $\left(-a_{1}\right.$, $\left.g_{1}\left(-a_{1} ; a_{4}, \eta_{2}\left(a_{4}\right)\right)\right)$; se la curva di equazione $\left(32_{1}\right)$ non incontra la curva $Y=\eta_{2}(X)$, incontra in un punto $H^{111}$ la curva di equazione $\left(27_{1}\right)$. Il campo $\Delta$ è limitato nella parte corrispondente a $Y \geqslant \eta_{1}(X),\left(-a_{1} \leqslant X \leqslant a_{2}\right)$ da archi delle curve $\left(30_{1}\right)$, (31) oppure $\left(30_{1}\right),\left(27_{2}\right)$ (cioè dagli archi $A_{1} M$ e $M K$ oppure $A_{1} K^{[1]}$ e $K^{[11} B_{2}$ ), e nella parte corrispondente a $Y \leqslant \eta_{2}(X),\left(-a_{1} \leqslant X \leqslant a_{2}\right)$ da archi delle curve $\left(32_{2}\right)$, (33) oppure $\left(32_{2}\right),\left(2 \pi_{1}\right)$ (cioè dagli archi $H N$ e $N B_{1}$ oppure $A_{2} H^{[11}$ e $\left.H^{[1]} B_{1}\right)\left({ }^{18}\right.$ bis)

(18) Il caso $k=2$ è stato considerato in 1).

$\left.{ }^{18 \mathrm{big}}\right)$ Si lascia al lettore di tracciare la figura. 
e) In ognuno dei casi esaminati in $d$ ), il campo $\Delta$ appartiene al campo $\Delta^{[1]}$ (in 1) coincide con $\Delta^{(11)}$. Inoltre considerazioni, fatte direttamente caso per caso, assicurano che, se $(x, y)$ è un punto qualsiasi del campo $A$, la curva $Y=g_{r}(X ; x, y)$, $(r=2, \ldots, \mu)$ ineontra la curva $Y=\eta_{1}(X)$ in un punto $X=\psi_{r}(x, y)$, dove $\psi_{r}(x, y)$ è definito dalla

$$
g_{r}\left(\psi_{r}(x, y) ; x, y\right)=\eta_{1}\left(\psi_{r}(x, y)\right), \quad(r=2, \ldots, \mu)
$$

i punti $\left(X, g_{r}(X ; x, y)\right)$, corrispondenti a valori di $X$, compresi tra $\psi(x, y)$ e $x$, appartengono al campo $\Delta$; tenuto conto della $\left(28_{1}\right)$ (cfr. capoverso $\left.c\right)$ ) è $\psi_{2}(x, y)=\psi(x, y)$. Inoltre, se $(x, y)$ è un punto qualsiasi del campo $A$, la curva $Y=g_{1}(X ; x, y),\left(-a_{1} \leqslant\right.$ $\left.\leqslant X \leqslant a_{2}\right)$ incontra la curva $Y=\chi(X)$ in un punto di ascissa $X=\chi(x, y)$, definita dalla $\left(28_{2}\right)$, e i punti $\left(X, g_{1}(X ; x, y)\right)$ corrispondenti a valori di $X$, compresi tra $\chi(x, y)$ e $x$, appartengono al campo $\Delta$.

f) Assunte di nuovo $(x, y)$ come coordinate correnti nel campo $A^{\mathrm{fl1}}$, si faccia in esso il cambiamento di variabili

$$
u=\psi(x, y), \quad v=\chi(x, y) \quad\left((x, y) \text { in } \Delta^{[11}\right),
$$

il quale, in base a quanto rilevato nel capoverso $c$ ), stabilisce una corrispondenza biunivoca tra il eampo $\Delta^{[1]}$ del piano $(x, y)$ e un campo $\delta^{[1]}$ del piano $(u, v)\left({ }^{19}\right)$; nei punti della curva $y=\eta_{1}(x),\left(-a_{1} \leqslant x \leqslant a_{2}\right)$ è $u=x, v=0$, e nei punti della curva $y=\eta_{2}(x),\left(-a_{1} \leqslant x \leqslant a_{2}\right)$ ѐ $u=0, v=x$.

In virtù delle $(29)$, le funzioni $\psi(x, y), \chi(x, y)$ sono continue in $\Delta^{\text {[1] }}$ assieme alle loro derivate prime e risulta

$$
J\left(\begin{array}{ll}
u & v \\
x & y
\end{array}\right)=\frac{\exp \left[I_{1}(\chi(x, y) ; x, y)\right] \exp \left[I_{2}(\psi(x, y) ; x, y)\right]\left[\sigma_{2}(x, y)-\sigma_{1}(x, y)\right]}{\left[\sigma_{2}\left(\psi(x, y), \eta_{1}(\psi(x, y))\right)-\sigma_{1}(\ldots)\right]\left[\sigma_{2}\left(\chi(x, y), \eta_{2}(\chi(x, y))\right)-\sigma_{1}(\ldots)\right]}
$$

In tutto il campo $\Delta^{\mathrm{I11}}$ è dunque

$$
J\left(\begin{array}{ll}
u & v \\
x & y
\end{array}\right) \neq 0
$$

e dalle (35) si possono ottenere le

$$
x=\xi(u, v), \quad y=\eta(u, v), \quad\left((u, v) \text { in } \delta^{[1]}\right),
$$

( $\left.{ }^{19}\right)$ Il campo $\delta^{[1]}$ del piano $(u, v)$ è limitato dai segmenti $u=-a_{1}, 0 \leqslant v \leqslant a_{2} ;-a_{1} \leqslant u \leqslant 0$, $v=a_{2} ; 0 \leqslant u \leqslant a_{2}, v=-a_{1} ; u=a_{2},-a_{1} \leqslant v \leqslant 0$, e dagli archi di eurva, definiti in forma parametrica (assumendo $y$ come parametro)

$$
\begin{aligned}
& u=\psi\left(-a_{1}, y\right), \quad v=\chi\left(-a_{1}, y\right), \quad\left(\eta_{\mathrm{a}}\left(-a_{1}\right) \leqslant y \leqslant \eta_{1}\left(-a_{1}\right)\right), \\
& u=\psi\left(a_{2}, y\right), \quad v=\chi\left(a_{2}, y\right), \quad\left(\eta_{1}\left(a_{2}\right) \leqslant y \leqslant \eta_{2}\left(a_{2}\right)\right) ;
\end{aligned}
$$

dalle prime due equazioni si ricava $v=v_{1}(u),\left(-a_{1} \leqslant u \leqslant 0\right)$, e dalle altre due $v=v_{2}(u)$, $\left(0 \leqslant u \leqslant a_{2}\right)$, dove le funzioni $v_{1}(u), v_{2}(u)$ sono decrescenti nei rispettivi intervalli di definizione. Si lascia al lettore il tracciare la semplice figura. 
dove le funzioni $\xi(u, v), \eta(u, v)$ sono continue assieme alle loro derivate prime nel campo $\delta^{\text {[u] }}$, ed è

$$
\xi(u, 0)=u, \quad \eta(u, 0)=\eta_{1}(u), \quad \xi(0, v)=v, \quad \eta(0, v)=\eta_{2}(v)
$$

Al campo $\Delta$ appartenente al campo $\Delta^{11}$ le $(35),\left(35_{1}\right)$ fanno corrispondere biunivocamente un campo $\delta$ appartenente a $\delta^{[1]}$.

g) Considerato l'integrale (1) del sistema (I) soddisfacente le condizioni del teorema, si ponga

$$
\zeta_{i}(u, v)=z_{i}(\xi(u, v), \eta(u, v)), \quad(i=1, \ldots, m) .
$$

Le funzioni $z_{i}(x, y),(i=1, \ldots, m)$ sono, per ipotesi, lipschitziane nel complesso delle variabili $(x, y)$ in tutto il campo $\Delta$, e quindi sono differenziabili in quasi tutto il campo $\Delta\left({ }^{20}\right)$; in virtù delle (36), (37), ad un insieme di misura nulla del campo $\Delta$ le $(35),\left(35_{1}\right)$ fanno corrispondere un insieme di misura nulla del campo $\delta\left({ }^{21}\right)$.

In quasi tutti $i$ punti del campo $\delta$ esistono allora le derivate

$$
\frac{\partial \zeta_{i}(u, v)}{\partial u}, \quad \frac{\partial \zeta_{i}(u, v)}{\partial v},
$$

le quali sono limitate nel campo $\delta$, ed inoltre valgono le

$$
\begin{aligned}
& \frac{\partial \zeta_{i}(u, v)}{\partial u}=p_{i}(\xi(u, v), \eta(u, v)) \frac{\partial \xi(u, v)}{\partial u}+q_{i}(\ldots) \frac{\partial \eta(u, v)}{\partial u}, \\
& \frac{\partial \zeta_{i}(u, v)}{\partial v}=p_{i}(\xi(u, v), \eta(u, v)) \frac{\partial \xi(u, v)}{\partial v}+q_{i}(\ldots) \frac{\partial \eta(u, v)}{\partial v} .
\end{aligned}
$$

Tenuto conto delle (29) e delle posizioni (4), il cambiamento di variabili (35), $\left(35_{1}\right)$ muta il sistema (I) nel sistema

$$
\left\{\begin{array}{rr}
\sum_{j=1}^{m} a_{i j}(u, v) \frac{\partial \zeta_{j}(u, v)}{\partial u}=F_{i}\left(u, v ; \zeta_{i}(u, v), \ldots, \zeta_{m}(u, v)\right), & \left(i=1, \ldots, v_{1}\right), \\
\sum_{j=1}^{m} a_{i j}(u, v) \frac{\partial \zeta_{j}(u, v)}{\partial v}=F_{i}\left(u, v ; \zeta_{1}(u, v), \ldots, \zeta_{m}(u, v)\right), & \left(i=v_{1}+1, \ldots, v_{1}+v_{2}\right), \\
\sum_{j=1}^{m} a_{i j}(u, v)\left(\frac{\partial \zeta_{j}(u, v)}{\partial u}+R_{i}(u, v) \frac{\partial \zeta_{j}(u, v)}{\partial v}\right)=F_{i}\left(u, v ; \zeta_{1}(u, v), \ldots, \zeta_{m}(u, v)\right), & \left(i=v_{1}+v_{2}+1, \ldots, m\right),
\end{array}\right.
$$

$\left.{ }^{20}\right)$ Cfr. H. Rademacher [1], Parte I, n. 3, Teorema I, p. 347.

${ }^{21}$ Cfr. H. Rademacher [1], Parte I, n. 7, pp. 354-355; n. 9, p. 359.

13 - Annali di Matematica 
dove si è posto

$$
\begin{gathered}
a_{i j}(u, v)=A_{i j}(\xi(u, v), \eta(u, v)), \quad(i, j=1, \ldots, m) . \\
R_{i}(u, v)=\left[\frac{\chi_{x}(x, y)+\varrho_{i}(x, y) \chi_{y}(x, y)}{\psi_{x}(x, y)+\varrho_{i}(x, y) \psi_{y}(x, y)}\right]_{x=\xi(u, v), y=\eta(u, v)}, \\
\left(i=v_{1}+v_{2}+1, \ldots, m\right), \\
\left\{\begin{array}{c}
F_{i}\left(u, v ; \zeta_{1}, \ldots, \zeta_{m}\right)=\left[\frac{f_{i}\left(x, y ; z_{1}, \ldots, z_{m}\right)}{\psi_{x}(x, y)+\varrho_{i}(x, y) \psi_{y}(x, y)}\right]_{x=\xi(u, v), y=\eta(u, v)}, \\
\left(i=1, \ldots, v_{1} ; v_{1}+v_{2}+1, \ldots, m\right), \\
F_{i}\left(u, v ; \zeta_{1}, \ldots, \zeta_{m}\right)=\left[\frac{f_{i}\left(x, y ; z_{1}, \ldots, z_{m}\right)}{\chi_{x}(x, y)+\varrho_{i}(x, y) \chi_{y}(x, y)}\right]_{x=\xi(u, v), y=\eta(u, v)}, \\
\left(i=v_{1}+1, \ldots, v_{1}+v_{2}\right) .
\end{array}\right.
\end{gathered}
$$

Le funzioni $a_{i j}(u, v), R_{i}(u, v)$ sono definite nel campo $\delta^{[1]}$, e le funzioni $F_{i}(u, v$; $\left.\zeta_{1}, \ldots, \zeta_{m}\right)$ sono definite per $(u, v)$ nel campo $\delta^{(11}$ e per tutte le $m$-ple $\left(\zeta_{1}, \ldots, \zeta_{m}\right)$ reali. Le funzioni (38) soddisfano il sistema (39) quasi ovunque nel campo $\delta$. Nelle $\left(40_{2}\right)$ sono tenute presenti le posizioni (4); in virtù di queste ultime possiamo porre

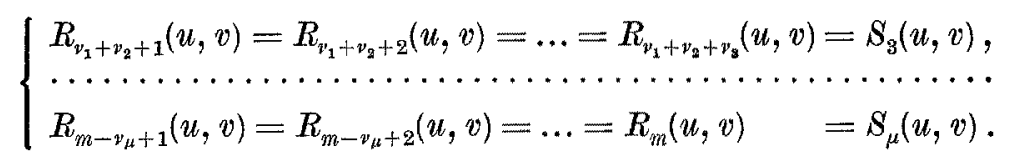

Inoltre, in virtù delle ipotesi del teorema e di quanto è stato rilevato nei capoversi $e), f)$, le funzioni $a_{i j}(u, v),(i, j=1, \ldots, m)$ sono lipschitziane nel complesso delle variabili nel campo $\delta^{[1]}\left({ }^{22}\right)$.

Le condizioni (19), (20) divengono rispettivamente

$$
\begin{aligned}
& \zeta_{j}(u, 0)=Z_{j}(u), \quad\left(-a_{1} \leqslant u \leqslant a_{2}\right) \quad \text { per } j=1, \ldots, m \\
& \sum_{j=1}^{m} b_{i j}(v) \zeta_{j}(0, v)=G_{i}(v), \quad\left(i=1, \ldots, v_{1}\right) .
\end{aligned}
$$

h) Indicate con $(X, Y)$ le coordinate correnti nel campo $\Delta$ e con $(\lambda, \mu)$ le corrispondenti coordinate nel campo $\delta$, così che

$$
\lambda=\psi(X, Y), \quad \mu=\chi(X, Y)
$$

(22) Le funzioni $A_{i j}(x, y)$ sono lipschitziane nel complesso delle variabili nel campo $D_{\infty}$; le funzioni $\left(35_{1}\right)$ hanno derivate parziali prime continue nel campo chiuso $\delta^{[1 ;}$; tenuto conto anche della forma di tale campo (cfr. nota $\left({ }^{19}\right)$ ), ne segue che le funzioni $a_{i j}(u$, $v$ ), definite dalle $\left(40_{1}\right)$, sono lipschitziane nel complesso delle variabili nel campo $\delta^{[1]}$. 
se $(x, y)$ è un punto fissato in $A, \mathrm{e}(u, v)$ il punto corrispondente di $\delta$, all'arco di curva $X=g_{1}(X ; x, y)$, ottenuto per valori di $X$, compresi tra $\chi(x, y)$ e $x$ corrisponde in $\delta$ il segmento $\mu=v ; 0 \leqslant \lambda \leqslant u$; all'arco della curva $Y=g_{2}(X ; x, y)$, ottenuto per valori di $X$, compresi tra $\psi(x, y)$ e $x$, corrisponde in $\delta$ il segmento $\lambda=u, 0 \leqslant \mu \leqslant v$; in corrispondenza ad ogni intiero $i$ (con $\left.i=v_{1}+v_{2}+1, \ldots, m\right)\left({ }^{23}\right)$, all'arco della curva

$$
Y=g_{i}(X ; x, y)
$$

ottenuto per valori di $X$ compresi tra $\psi_{i}(x, y)$ e $x$ corrisponde in $\delta$ un areo della curva, definita dalle equazioni parametriche

$$
\lambda=\psi\left(X, g_{i}(X ; x, y)\right), \quad \mu=\chi\left(X, g_{i}(X ; x, y)\right)
$$

nel parametro $X$ con $X$ compreso tra $\psi_{i}(x, y)$ e $x$.

Dalle (43) segue

$$
\begin{aligned}
& \frac{d \lambda}{d X}=\psi_{X}\left(X, g_{i}(X ; x, y)\right)+\psi_{Y}(\ldots) \varrho_{i}(X ; x, y), \\
& \frac{d \mu}{d X}=\chi_{X}\left(X, g_{i}(X ; x, y)\right)+\chi_{P}(\ldots) \varrho_{i}(X ; x, y) ;
\end{aligned}
$$

tenuto conto delle (29) (nelle quali si ponga $\left(X, g_{i}(X ; x, y)\right)$ al posto di $\left.(x, y)\right)$ e delle (4), si verifica immediatamente che $d \lambda / d X \neq 0, d \mu / d X \neq 0$; quindi dalle (43), tenuto conto anche delle $\left(35_{1}\right)$, si ottiene l'equazione dell'arco di curva corrispondente nel campo $\delta$ all'arco considerato della curva (42) del campo $\Delta$ nella forma

$$
\mu=\gamma_{i}(\lambda ; u, v)
$$

0 anche

$$
\lambda=\gamma_{i}^{*}(\mu ; u, v) .
$$

Al punto $\left(\psi_{i}(x, y), \eta_{1}\left(\psi_{i}(x, y)\right)\right)$ della curva $\gamma_{1}$ corrisponde il punto $\left(\gamma_{i}^{*}(0 ; u, v), 0\right)$ del segmento $-a_{1} \leqslant \lambda \leqslant a_{2}, \mu=0$; per brevità poniamo

$$
\gamma_{i}^{*}(0 ; u, v)=\varphi_{i}(u, v)
$$

${ }^{(23)}$ In conformità alle notazioni usate finora si fanno assumere $a i$ i valori $v_{1}+v_{2}+1, \ldots, m$; tenuto conto delle (4) risulta

$$
\begin{aligned}
g_{v_{1}+v_{2}+1}(X ; x, y) & =g_{v_{1}+v_{2}+2}(X ; x, y)=\ldots= \\
= & g_{v_{1}+v_{2}+v_{3}}(X ; x, y), \ldots, g_{m-v_{\mu}+1}(X ; x, y)=g_{m-v_{\mu}+2}(X ; x, y)=\ldots=g_{m}(X ; x, y) .
\end{aligned}
$$


così che $\gamma_{i}\left(\varphi_{i}(u, v) ; u, v\right)=0$. La funzione $\gamma_{i}(\lambda ; u, v)$ è continua assieme alla sua derivata prima rispetto a $\lambda$, e risulta

$$
\frac{d \gamma_{i}(\lambda ; u, v)}{d \lambda}=R_{i}\left(\lambda, \gamma_{i}(\lambda ; u, v)\right)
$$

i) Nelle prime $\nu_{1}$ tra le (39) si ponga $\lambda$ al posto di $u$ e si integri tra zero e $u$; si ottengono così le

$$
\sum_{j=1}^{m} \int_{0}^{u} a_{i j}(\lambda, v) \frac{\partial \zeta_{j}(\lambda, v)}{\partial \lambda} d \lambda=\int_{0}^{u} F_{i}\left(\lambda, v ; \zeta_{1}(\lambda, v), \ldots, \zeta_{m}(\lambda, v)\right) d \lambda, \quad\left(i=1, \ldots, v_{1}\right)
$$

e con una integrazione per parti $\left({ }^{23 \text { bis }}\right)$

$$
\begin{aligned}
& \sum_{j=1}^{m} a_{i j}(u, v) \zeta_{j}(u, v)=\sum_{j=1}^{m} a_{i j}(0, v) \zeta_{j}(0, v)+ \\
& +\sum_{j=1}^{m} \int_{0}^{u} \frac{\partial a_{i j}(\lambda, v)}{\partial \lambda} \zeta_{j}(\lambda, v) d \lambda+\int_{0}^{u} F_{i}\left(\lambda, v ; \zeta_{1}(\lambda, v), \ldots, \zeta_{m}(\lambda, v)\right) d \lambda, \quad\left(i=1, \ldots, v_{1}\right) .
\end{aligned}
$$

Le $\left(47_{1}\right)$ per quasi tutti i valori $v$, tali che alle rette $v=$ cost. appartengano punti del campo $\delta$, valgono per ogni $u$, con $(u, v)$ appartenente al campo $\delta$. Posto nelle successive $v_{2}$ equazioni (39) $\mu$ al posto di $v$, integrando tra zero e $v$, si ottengono le

$$
\sum_{j=1}^{m} \int_{0}^{v} a_{i j}(u, \mu) \frac{\partial \zeta_{j}(u, \mu)}{\partial \mu} d \mu=\int_{0}^{v} F_{i}\left(u, \mu ; \zeta_{1}(u, \mu), \ldots, \zeta_{m}(u, \mu)\right) d \mu, \quad\left(i=v_{1}+1, \ldots, \nu_{2}\right),
$$

e con una integrazione per parti, tenendo conto delle (19),

$$
\begin{aligned}
\sum_{j=1}^{m} a_{i j}(u, v) \zeta_{j}(u, v) & =\sum_{j=1}^{m} a_{i j}(u, 0) Z_{j}(u)+\sum_{j=1}^{m} \int_{0}^{v} \frac{\partial a_{i j}(u, \mu)}{\partial \mu} \zeta_{j}(u, \mu) d \mu+ \\
& +\int_{0}^{v} F_{i}\left(u, \mu ; \zeta_{1}(u, \mu), \ldots, \zeta_{m}(u, \mu)\right) d \mu, \quad\left(i=v_{1}+1, \ldots, v_{1}+v_{2}\right) .
\end{aligned}
$$

Le $\left(47_{2}\right)$, in corrispondenza a quasi tutti gli $u,\left(-a_{1} \leqslant u \leqslant a_{2}\right)$ valgono per ogni $v$, tale che il punto $(u, v)$ appartenga al campo $\delta$.

(23bis) Le funzioni $a_{i j}(u, v)$ sono lipschitziane nel complesso delle variabili nel campo $\delta^{[1]}$ (cfr. nota $\left({ }^{22}\right)$ ); è quindi lecita l'integrazione per parti; osservazione analoga a proposito (più avanti) delle $\left(47_{2}\right)$. 
Poichè le funzioni $z_{j}(\lambda, \mu)=z_{j}(\xi(\lambda, \mu), \eta(\lambda, \mu))$ sono lipschitziane nel complesso delle variabili $(\lambda, \mu)$ nel campo $\left.\delta{ }^{24}\right)$, esistono $m$ costanti $K_{j},(j=1, \ldots, m)$, tali che

$$
\left|\zeta_{j}(\lambda, \mu)-\zeta_{j}(\bar{\lambda}, \bar{\mu})\right| \leqslant K_{j}\{|\lambda-\bar{\lambda}|+|\mu-\bar{\mu}|\}, \quad(j=1, \ldots, m)
$$

per ogni coppia di punti $(\lambda, \mu),(\bar{\lambda}, \bar{\mu})$ del campo $\delta$.

Tenuto conto delle $\left(40_{2}\right)$, le funzioni $R_{i}(\lambda, \mu),\left(i=v_{1}+v_{2}+1, \ldots, m\right)$ sono continue e mai nulle in tutto il campo $\delta^{[1]}$; esistono quindi due costanti positive $k$ e $M^{[1]}$, tali che in tutto il campo $\delta^{[1]}$ sia

$$
1 / k \leqslant\left|R_{i}(\lambda, \mu)\right| \leqslant M^{[1]}, \quad\left(i=v_{1}+\nu_{2}+1, \ldots, m\right) ;
$$

in virtù delle (46) e (48) risulta allora

$$
\begin{aligned}
\left|\zeta_{j}\left(\lambda, \gamma_{i}(\lambda ; u, v)\right)-\zeta_{j}\left(\bar{\lambda}, \gamma_{i}(\bar{\lambda} ; u, v)\right)\right| \leqslant K_{j}\left(1+M^{[1]}\right)|\lambda-\bar{\lambda}| & \\
& \left(j=1, \ldots, m ; i=v_{1}+v_{2}+1, \ldots, m\right) .
\end{aligned}
$$

In quasi tutti i punti della curva $\mu=\gamma_{i}(\lambda ; u, v)$ appartenenti al campo $\delta$ esiste dunque la derivata

$$
\frac{d \zeta_{j}\left(\lambda, \gamma_{i}(\lambda ; u, v)\right)}{d \lambda}, \quad\left(j=1, \ldots, m ; i=v_{1}+v_{2}+1, \ldots, m\right)
$$

Se $i$ è uno tra i numeri $\nu_{1}+\nu_{2}+1, \ldots, m$, posto $w=\varphi_{i}(u, v)$, l'equazione della curva $\mu=\gamma_{i}(\lambda ; u, v)$, tenuto conto della (45), può essere scritta nella forma

$$
\mu=\gamma_{i}(\lambda ; w, 0), \quad\left(-a_{1} \leqslant w \leqslant a_{2}\right)
$$

Si prova facilmente $\left(^{25}\right)$ che, in corrispondenza a quasi tutti i valori $w$ di $\left(-a_{1}, a_{2}\right)$, per quasi tutti i valori di $\lambda$, compresi tra $w$ e $u$ è

$$
\frac{d \zeta_{j}\left(\lambda, \gamma_{i}(\lambda ; u, v)\right)}{d \lambda}=\frac{\partial \zeta_{j}\left(\lambda, \gamma_{i}(\lambda ; u, v)\right)}{\partial \lambda}+R_{i}\left(\lambda, \gamma_{i}(\lambda ; u, v)\right) \frac{\partial \zeta_{j}\left(\lambda, \gamma_{i}(\lambda ; u, v)\right)}{\partial \mu}
$$

${ }^{(24)}$ Per ipotesi le funzioni $z_{j}(x, y),(j=1, \ldots, m)$ sono lipschitziane nel complesso delle variabili nel campo 4 ; poichè le funzioni $\left(35_{1}\right)$ hanno derivate parziali continue nel campo chiuso $\delta^{[1]}$ (a cui appartiene $\delta$ ), tenuto anche conto della configurazione del campo $\delta$ (che sarebbe agevole disegnare), segue che le funzioni (38) sono lipschitziane nel complesso dello variabili $(u, v)$ nel campo $\delta$; si fa presente che nel seguito del presente capoverso sono assunte $(\lambda, \mu)$ come coordinate correnti nel campo $\delta$, mentre $(u, v)$ è un punto fissato del campo stesso (efr. anche capoverso $h)$ ).

${ }^{(25)}$ Al variare di $w$ in $\left(-a_{1}, a_{2}\right)$ le curve $\left(44^{\prime \prime}\right)$ coprono tutto il campo $\delta$ (nel senso che per ogni punto di $\delta$ ne passa una e una sola); allora le

$$
\lambda=\lambda, \quad \mu=\gamma_{i}(\lambda ; w, 0),
$$


e inoltre vale la $i$-esima tra le equazioni (39), nella quale si assumano come coordinate correnti $\lambda, \mu$, e si ponga $\mu=\gamma_{i}(\lambda ; u, v)$; tale equazione può dunque essere scritta nella forma

$$
\sum_{j=1}^{m_{i}} a_{i j}\left(\lambda, \gamma_{i}(\lambda ; u, v)\right) \frac{d \zeta_{j}\left(\lambda, \gamma_{i}(\lambda ; u, v)\right)}{d \lambda}=F_{i}\left(\lambda, \gamma_{i}(\lambda ; u, v) ; \zeta_{1}\left(\lambda, \gamma_{i}(\lambda ; u, v)\right), \ldots, \zeta_{m}(\ldots)\right)
$$

Integrando rispetto a $\lambda$ tra $\varphi_{i}(u, v)$ e $u$ si ottiene la

$$
\begin{aligned}
\sum_{j=1}^{m} \int_{\varphi_{i}(u, v)}^{u} a_{i j}\left(\lambda, \gamma_{i}(\lambda ; u, v)\right) \frac{d \zeta_{1}\left(\lambda, \gamma_{i}(\lambda ; u, v)\right)}{d \lambda} d \lambda= \\
\quad=\int_{\varphi_{i}(u, v)}^{u} F_{i}\left(\lambda, \gamma_{i}(\lambda ; u, v) ; \zeta_{1}\left(\lambda, \gamma_{i}(\lambda ; u, v)\right), \ldots, \zeta_{m}(\ldots)\right) d \lambda,
\end{aligned}
$$

e con una integrazione per parti $\left({ }^{26}\right)$, tenendo conto delle $\left(19_{1}\right)$,

$$
\begin{aligned}
& \sum_{j=1}^{m} a_{i j}(u, v) \zeta_{j}(u, v)=\sum_{j=1}^{m} a_{i j}\left(\varphi_{i}(u, v), 0\right) Z_{j}\left(\varphi_{i}(u, v)\right)+ \\
+ & \sum_{j=1}^{m} \int_{p_{i}(u, v)}^{u} \frac{d a_{i j}\left(\lambda, \gamma_{i}(\lambda ; u, v)\right)}{d \lambda} \zeta_{j}\left(\lambda, \gamma_{i}(\lambda ; u, v)\right) d \lambda+ \\
+ & \int_{\varphi_{i}(u, v)}^{u} F_{i}\left(\lambda, \gamma_{i}(\lambda ; u, v) ; \zeta_{1}\left(\lambda, \gamma_{i}(\lambda ; u, v)\right), \ldots, \zeta_{m}(\ldots)\right) d \lambda, \quad\left(i=v_{1}+v_{2}+1, \ldots, m\right) .
\end{aligned}
$$

Le (47) valgono in quasi tutto il campo $\delta$; considerazioni di tipo elementare permettono di provare che il secondo membro di ognuna delle (47) è funzione continua

fanno corrispondere al campo $\delta$ del piano $(\lambda, \mu)$ un campo $T$ del piano $(\lambda, w)$; la corrispondenza è biunivoca, e, poichè vale la $(46)$, ed è $R_{i}(\lambda, \mu) \neq 0$ (efr. le $\left(40_{2}\right)$ ), si prova immediatamente che è $\partial \gamma_{i}(\lambda ; w, 0) / \partial w \neq 0$; è quindi $J\left(\begin{array}{ll}\lambda & \mu \\ \lambda & w\end{array}\right) \neq 0$, e (cfr. nota $\left.\left({ }^{21}\right)\right)$ ad un insieme di misura nulla del campo $\delta$ corrisponde biunivocamente un insieme di misura nulla del campo $T$; quindi le derivate $\partial \zeta_{j}(\lambda, \mu) / \partial \lambda, \partial \zeta_{j}(\lambda, \mu) / \partial \mu,(j=1, \ldots, m)$, nelle quali si ponga $\mu=p_{i}(\lambda ; w, 0)$, esistono in quasi tutti $\mathrm{i}$ punti del campo $T$, e soddisfano l'equazione $i$-esima tra le (39); ne segue che per quasi tutti i valori $w,\left(-a_{1} \leqslant w \leqslant a_{2}\right)$ tali derivate esistono in quasi tutti i punti della curva $\left(44^{\prime \prime}\right)$, vale la (51) (dove si tenga conto che $\gamma_{i}(\lambda ; u, v)=\gamma_{i}\left(\lambda ; p_{i}(u, v), 0\right)$ e si applichi un noto teorema di derivazione delle funzioni composte; cfr. G. Scorza DraGONI [1]), ed è soddisfatta la $i$-esima tra le (39).

$\left({ }^{26}\right)$ Essendo le $a_{i j}(\lambda, \mu)$ lipschitziane nel complesso delle variabili nel campo $\delta^{[1]}$, considerazioni del tutto analoghe a quelle sviluppate per stabilire le (50) permettono di provare che, fissato il punto $(u, v)$ in $\delta$, le funzioni $a_{i j}\left(\lambda, \gamma_{i}(\lambda ; u, v)\right)$ sono lipschitziane in $\lambda$ nell'intervallo $\left(\varphi_{i}(u, v), u\right)$; è quindi lecita l'integrazione per parti. 
nel complesso delle variabili $(u, v)$ in ogni punto del campo $\delta\left({ }^{27}\right)$, e, poichè il primo membro di ognuna delle (47) è pure funzione continua nel complesso delle variabili $(u, v)$, le $(47)$ valgono in tutto il campo $\delta$.

j) Dal sistema delle $m$ equazioni algebriche (47) lineari nelle $\zeta_{j}(u, v),(j=$ $=1, \ldots, m)$, tenuto conto della $(8)$ e delle $\left(40_{1}\right)$, e indicato con $\alpha_{i j}(u, v)$ il comple-

${ }^{(27)}$ Le ipotesi fatte, assieme alle $\left(40_{1}\right),\left(40_{3}\right)$, assicurano la continuità nel complesso delle variabili $(u, v)$ dei singoli termini, che compaiono a secondo membro delle (47), tranne per quanto riguarda $i$ termini rispettivi

$$
\int_{0}^{u} \frac{\partial a_{i j}(\lambda, v)}{\partial \lambda} \zeta_{j}(\lambda, v) d \lambda, \quad \int_{0}^{v} \frac{\partial a_{i j}(u, \mu)}{\partial \mu} \zeta_{j}(u, \mu) d \mu, \quad \int_{\varphi_{i}(u, v)}^{u} \frac{d a_{i j}\left(\lambda, \gamma_{i}(\lambda ; u, v)\right)}{d \lambda} \zeta_{j}\left(\lambda, \gamma_{i}(\lambda ; u, v)\right) d \lambda .
$$

Ora si ha, per esempio, supposto, per fissare le idee, che il punto $(u, v)$ sia interno al campo $\delta$,

$$
\begin{aligned}
& \left|\int_{0}^{u+h} \frac{\partial a_{i j}(\lambda, v+k)}{\partial \lambda} \zeta_{j}(\lambda, v+k) d \lambda-\int_{0}^{u} \frac{\partial a_{i j}(\lambda, v)}{\partial \lambda} \zeta_{j}(\lambda, v) d \lambda\right| \leqslant \\
& \quad \leqslant\left|\int_{0}^{u+h} \frac{\partial a_{i j}(\lambda, v+k)}{\partial \lambda} \zeta_{j}(\lambda, v+k) d \lambda\right|+\left|\int_{0}^{b} \frac{\partial\left[a_{i j}(\lambda, v+k)-a_{i j}(\lambda, v)\right]}{\partial \lambda} \zeta_{j}(\lambda, v+k) d \lambda\right|+ \\
& \quad+\left|\int_{0}^{u} \frac{\partial a_{i j}(\lambda, v)}{\partial \lambda}\left[\zeta_{j}(\lambda, v+k)-\zeta_{j}(\lambda, v)\right] d \lambda\right| .
\end{aligned}
$$

Tenuto conto che

$$
\begin{aligned}
& \left|\int_{0}^{u} \frac{\partial\left[a_{i j}(\lambda, v+k)-a_{i j}(\lambda, v)\right]}{\partial \lambda} \zeta_{j}(\lambda, v+k) d \lambda\right| \leqslant \\
& \quad \leqslant\left|a_{i j}(0, v+k)-a_{i j}(0, v)\right|\left|\zeta_{j}(0, v+k)\right|+\left|a_{i j}(\lambda, v+k)-a_{i j}(u, v)\right|\left|\zeta_{j}(u, v+k)\right|+ \\
& \quad+\left|\int_{0}^{u}\right| a_{i j}(\lambda, v+k)-a_{i j}(\lambda, v)||\left|\frac{d \zeta_{j}(\lambda, v+k)}{d \lambda}\right| d \lambda,
\end{aligned}
$$

e che le $a_{i j}(u, v), \zeta_{j}(u, v)$ sono lipschitziane nel campo $\delta$, segue immediatamente la continuità in $(u, v) \mathrm{di}$

$$
\int_{0}^{\imath k} \frac{\partial a_{i j}(\lambda, v)}{\partial \lambda} \zeta_{j}(\lambda, v) d \lambda
$$


mento algebrico delle $a_{i j}(u, v)$ nel determinante $A$ si ottengono le

$$
\begin{aligned}
z_{j}(u, v) & =\sum_{i=1}^{v_{1}} \alpha_{i j}(u, v)\left\{\sum_{s=1}^{m} a_{i s}(0, v) \zeta_{s}(0, v)+\right. \\
& \left.+\sum_{s=1}^{m} \int_{0}^{u} \frac{\partial a_{i s}(\lambda, v)}{\partial \lambda} \zeta_{s}(\lambda, v) d \lambda+\int_{0}^{u} F_{i}\left(\lambda, v ; \zeta_{1}(\lambda, v), \ldots, \zeta_{m}(\lambda, v)\right) d \lambda\right\}+ \\
& +\sum_{i=\gamma_{1}+1}^{\gamma_{1}+y_{z}} \alpha_{i j}(u, v)\left\{\sum_{s=1}^{m} a_{i s}(u, 0) Z_{s}(u)+\sum_{s=1}^{m} \int_{0}^{v} \frac{\partial a_{i s}(u, \mu)}{\partial \mu} \zeta_{s}(u, \mu) d \mu+\right. \\
& \left.+\int_{0}^{v} F_{i}\left(u, \mu ; \zeta_{1}(u, \mu), \ldots, \zeta_{m}(u, \mu)\right) d \mu\right\}+ \\
& +\sum_{i=v_{1}+v_{2}+1}^{m_{i}} \alpha_{i j}(u, v)\left\{\sum_{s=1}^{m} a_{i s}\left(\varphi_{i}(u, v), 0\right) Z_{s}\left(\varphi_{i}(u, v)\right)+\right. \\
& +\sum_{s=1}^{m} \int_{\varphi_{i}(u, v)}^{u} \frac{d a_{i s}\left(\lambda, \gamma_{i}(\lambda ; u, v)\right)}{d \lambda} \zeta_{s}\left(\lambda, \gamma_{i}(\lambda ; u, v)\right) d \lambda+ \\
& +\int_{\varphi_{i}(u, v)}^{u} F_{i}\left(\lambda, \gamma_{i}(\lambda ; u, v) ; \zeta_{1}\left(\lambda, \gamma_{i}(\lambda ; u, v)\right), \ldots, \zeta_{m}\left(\lambda, \gamma_{i}(\lambda ; u, v)\right)\right) d \lambda, \\
& (j=1, \ldots, m) .
\end{aligned}
$$

k) Le funzioni $\zeta_{j}(0, v),(j=1, \ldots, m)$ soddisfano le $\left(20_{1}\right)$; inoltre soddisfano le

$$
\begin{aligned}
\sum_{j=1}^{m} a_{i j}(0, v) \zeta_{j}(0, v) & =\sum_{j=1}^{m} a_{i j}(0,0) Z_{j}(0)+\sum_{j=1}^{m} \int_{0}^{v} \frac{\partial a_{i j}(0, \mu)}{\partial \mu} \zeta_{j}(0, \mu) d \mu+ \\
& +\int_{0}^{v} F_{i}\left(0, \mu ; \zeta_{j}(0, \mu), \ldots, \zeta_{m}(0, \mu)\right) d \mu, \quad\left(i=v_{1}+1, \ldots, v_{2}\right),
\end{aligned}
$$

ottenute dalle $\left(47_{2}\right)$ per $u=0$, e le

$$
\begin{aligned}
& \sum_{i=1}^{m_{i}} a_{i j}(0, v) \zeta_{j}(0, v)=\sum_{j=1}^{m_{m}} a_{i j}\left(\varphi_{i}(0, v), 0\right) Z_{j}\left(\varphi_{i}(0, v)\right)+ \\
+ & \sum_{j=1}^{m} \int_{\varphi_{i}(0, v)}^{0} \frac{d a_{i j}\left(\lambda, \gamma_{i}(\lambda ; 0, v)\right)}{d \lambda} \zeta_{j}\left(\lambda, \gamma_{i}(\lambda ; 0, v)\right) d \lambda+ \\
+ & \int_{\varphi_{i}(0, v)}^{0} F_{i}\left(\lambda, \gamma_{i}(\lambda ; 0, v) ; \zeta_{Y}\left(\lambda, \gamma_{i}(\lambda ; 0, v)\right), \ldots, \zeta_{m}(\ldots)\right) d \lambda, \quad\left(i=v_{1}+v_{2}+1, \ldots, m\right) .
\end{aligned}
$$


La (17), tenuto conto del cambiamento di variabili $(35),\left(35_{1}\right)$ e delle $\left(40_{1}\right)$, diviene

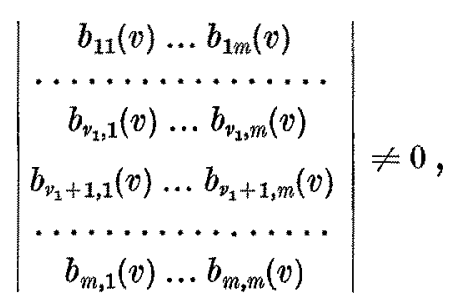

avendo posto, per uniformità di notazioni, $b_{i j}(v)=a_{i j}(0, v)$, per $i=v_{1}+1, \ldots, m$, $j=1, \ldots, m$. Indicato allora con $\beta_{i j}(v)$ il complemento algebrico di $b_{i j}(v)$ nel determinante $\left(17_{1}\right)$, diviso per il determinante stesso, dal sistema delle $m$ equazioni algebriche $\left(20_{1}\right),\left(20_{2}\right),\left(20_{i}\right)$ nelle incognite $z_{j}(0, v)$ si ottengono le

$$
\begin{aligned}
\zeta_{j}(0, v)= & \sum_{i=1}^{v_{1}} \beta_{i j}(v) G_{i}(v)+\sum_{i=v_{1}+1}^{v_{1}+y_{2}} \beta_{i j}(v)\left\{\sum_{s=1}^{m} a_{i s}(0, s) Z_{s}(0)+\right. \\
& \left.+\sum_{s=1}^{m} \int_{0}^{v} \frac{\partial a_{i s}(0, \mu)}{\partial \mu} \zeta_{s}(0, \mu) d \mu+\int_{0}^{v} F_{i}\left(0, \mu ; \zeta_{1}(0, \mu), \ldots, \zeta_{m}(0, \mu)\right) d \mu\right\}+ \\
& +\sum_{i=y_{1}+v_{2}+1}^{m} \beta_{i j}(v)\left\{\sum_{s=1}^{m} a_{i s}(\varphi,(0, v), 0) Z_{s}\left(\varphi_{i}(0, v)\right)+\right. \\
& +\sum_{s=1}^{m} \int_{\varphi_{i}(0, v)}^{0} \frac{d a_{i s}\left(\lambda, \gamma_{i}(\lambda ; 0, v)\right)}{d \lambda} \zeta_{s}\left(\lambda, \gamma_{i}(\lambda ; 0, v)\right) d \lambda+ \\
& \left.+\int_{\varphi_{i}(0, v)}^{0} F_{i}\left(\lambda, \gamma_{i}(\lambda ; 0, v) ; \zeta_{1}\left(\lambda, \gamma_{i}(\lambda ; 0, v)\right), \ldots, \zeta_{m}(\ldots)\right) d \lambda\right\}
\end{aligned}
$$

Le (53) valgono in un opportuno intervallo $-a_{1}^{\prime} \leqslant v \leqslant a_{2}^{\prime}\left(\operatorname{con} 0<a_{1}^{\prime} \leqslant a_{1}, 0<a_{2}^{\prime} \leqslant\right.$ $\left.\leqslant a_{2}\right)$, tale che i punti $(0, v)$ appartengano al campo $\delta$.

l) Sia ora

$$
z_{i}=z_{i}^{*}(x, y), \quad(i=1, \ldots, m)
$$

una $m$-pla di funzioni lipschitziane nel campo 4 , ivi soddisfacenti il sistema (I) quasi ovunque, e soddisfacente le

$$
z_{i}^{*}\left(x, \eta_{1}(x)\right)=Z_{i}(x), \quad(i=1, \ldots, m), \text { per }-a_{1} \leqslant x \leqslant a_{2},
$$

e le

$$
\sum_{j=1}^{m} b_{i j}(x) z_{i}^{*}\left(x, \eta_{2}(x)\right)=G_{i}(x), \quad\left(i=1, \ldots, v_{1}\right)
$$

nei punti della curva $y=\eta_{2}(x)$ appartenenti al campo $\Delta$. 
Posto

$$
\xi_{i}^{*}(u, v)=z_{i}^{*}(\xi(u, v), \eta(u, v)), \quad(i=1, \ldots, m)
$$

le funzioni $\zeta_{i}^{*}(u, v),(i=1, \ldots, m)$ soddisfano relazioni del tutto analoghe alle (52), (53); sottraendo tali relazioni (che, per brevità, sono omesse) dalle corrispondenti relazioni (52), (53) e ponendo

$$
U_{i}(u, v)=\zeta_{i}(u, v)-\zeta_{i}^{*}(u, v), \quad(i=1, \ldots, m)
$$

si ottengono le

$$
\begin{aligned}
& U_{j}(u, v)=\sum_{i=1}^{v_{1}} \alpha_{i j}(u, v)\left\{\sum_{s=1}^{m} a_{i s}(0, v) U_{s}(0, v)+\sum_{s=1}^{m} \int_{0}^{u} \frac{\partial a_{i s}(\lambda, v)}{\partial \lambda} U_{s}(\lambda, v) d \lambda+\right. \\
& \left.+\int_{0}^{u}\left[F_{i}\left(\lambda, v ; \zeta_{1}(\lambda, v), \ldots, \zeta_{m}(\lambda, v)\right)-F_{i}\left(\lambda, v ; \zeta_{1}^{*}(\lambda, v), \ldots, \zeta_{m}^{*}(\lambda, v)\right)\right] d \lambda\right\}+ \\
& +\sum_{i=y_{1}+1}^{v_{1}+y_{2}} \alpha_{i j}(u, v)\left\{\sum_{\delta=1}^{m} \int_{0}^{n} \frac{\partial a_{i s}(u, \mu)}{\partial \mu} U_{s}(u, \mu) d \mu+\right. \\
& \left.+\int_{0}^{v}\left[F_{i}\left(u, \mu ; \zeta_{1}(u, \mu), \ldots, \zeta_{m}(u, \mu)\right)-F_{i}\left(u, \mu ; \zeta_{1}^{*}(u, \mu), \ldots, \zeta_{m}^{*}(u, \mu)\right)\right] d \mu\right\}+ \\
& +\sum_{i=v_{1}+v_{2}+1}^{m} \alpha_{i j}(u, v)\left\{\sum_{s=1}^{m} \int_{\varphi_{i}(u, v)}^{u_{u}} \frac{d a_{i s}\left(\lambda, \gamma_{i}(\lambda ; u, v)\right)}{d \lambda} U_{s}\left(\lambda, \gamma_{i}(\lambda ; u, v)\right) d \lambda+\right. \\
& +\int_{\varphi_{i}(u, v)}^{u}\left[F_{i}\left(\lambda, \gamma_{i}(\lambda ; u, v) ; \zeta_{1}\left(\lambda, \gamma_{i}(\lambda ; u, v)\right), \ldots, \zeta_{m}(\ldots)\right)-\right. \\
& \left.\left.-F_{i}\left(\lambda, \gamma_{i}(\lambda ; u, v) ; \zeta_{i}^{*}\left(\lambda, \gamma_{i}(\lambda ; u, v)\right), \ldots, \zeta_{m}^{*}(\ldots)\right)\right] d \lambda\right\}, \quad(j=1, \ldots, m) .
\end{aligned}
$$

$$
\begin{aligned}
U_{j}(0, v) & =\sum_{i=\nu_{1}+1}^{v_{1}+p_{2}} \beta_{i j}(v)\left\{\sum_{s=1}^{m} \int_{0}^{v} \frac{\partial a_{i s}(0, \mu)}{\partial \mu} U_{s}(0, \mu) d \mu+\right. \\
& \left.+\int_{0}^{v}\left[F_{i}\left(0, \mu ; \zeta_{1}(0, \mu), \ldots, \zeta_{m}(0, \mu)\right)-F_{i}\left(0, \mu ; \zeta_{1}^{*}(0, \mu), \ldots, \zeta_{m}^{*}(0, \mu)\right)\right] d \mu\right\}+ \\
& +\sum_{i=v_{1}+v_{2}+1}^{m} \beta_{i j}(v)\left\{\sum_{s=1}^{m} \int_{\varphi_{i}(0, v)}^{0} \frac{d a_{i s}\left(\lambda, \gamma_{i}(\lambda ; 0, v)\right)}{d \lambda} U_{s}\left(\lambda, \gamma_{i}(\lambda ; 0, v)\right) d \lambda+\right. \\
& +\int_{\phi_{i}(0, v)}^{v}\left[F_{i}\left(\lambda, \gamma_{i}(\lambda ; 0, v)\right) ; \zeta_{1}\left(\lambda, \gamma_{i}(\lambda ; 0, v), \ldots, \zeta_{m}(\ldots)\right)-\right.
\end{aligned}
$$


Si indichino con $H, H_{1}, H_{2}$ costanti non negative, tali che in tutto il campo chiuso e limitato $\delta^{[13}$ sia

$$
\left|a_{i j}(u, v)\right| \leqslant H, \quad\left|\alpha_{i j}(u, v)\right| \leqslant H_{1}, \quad\left|\beta_{i j}(v)\right| \leqslant H_{2}, \quad(i, j=1, \ldots, m),
$$

costanti certo esistenti, per la continuità delle funzioni a primo membro delle (57) nel campo chiuso e limitato $\delta^{[1]}$.

Poichè le funzioni $a_{i j}(u, v)$ sono lipschitziane in $\delta^{[1]}$ ( $\mathrm{frr}$. nota $\left.{ }^{22}\right)$ ), si indichi con $A$ la loro costante di Lipschitz; dalle (13) e $\left(40_{3}\right)$ segue che le funzioni $F_{i}\left(u, v ; \zeta_{1}, \ldots, \zeta_{m}\right)$ sono lipschitziane nel complesso delle $\left(\zeta_{1}, \ldots, \zeta_{m}\right)$; si indichi con $A_{1}$ la costante di Lipschitz corrispondente.

Posto

$$
\Lambda+\Lambda_{\mathbf{1}}=L_{2}
$$

tenuto conto delle $(46),(49)$, dalle $(55),(56)$ si ottengono le $\left({ }^{28}\right)$

$$
\begin{aligned}
& \left|U_{j}(u, v)\right| \leqslant H_{1}\left\{v_{1} H \sum_{s=1}^{m}\left|U_{s}(0, v)\right|+\nu_{1} L_{2}\left|\int_{0}^{n} \sum_{s=1}^{m}\right| U_{s}(\lambda, v)|d \lambda|+\right. \\
& +v_{2} L_{2}\left|\int_{0}^{v} \sum_{s=1}^{m_{1}}\right| U_{s}(u, \mu)|d \mu|+ \\
& \left.+\left(L_{2}+\Lambda M^{[1]}\right) \sum_{i=\nu_{1}+v_{3}+1}^{m}\left|\int_{q_{t}(u, v)}^{u} \sum_{s=1}^{m}\right| U_{s}\left(\lambda, \gamma_{i}(\lambda ; u, v)\right)|d \lambda|\right\}, \quad(j=1, \ldots, m) . \\
& \left|U_{j}(0, v)\right| \leqslant H_{2}\left\{v_{2} L_{2}\left|\int_{0}^{b} \sum_{s=1}^{m}\right| U_{s}(0, \mu)|d \mu|+\right. \\
& \left.+\left(L_{2}+A M^{[11}\right) \sum_{i=v_{1}+v_{2}+1}^{m}\left|\int_{p_{i}(0, v)}^{0} \sum_{s=1}^{m}\right| U_{s}\left(\lambda, \gamma_{i}(\lambda ; 0, v)\right)|d \lambda|\right\}, \quad(j=1, \ldots, m) .
\end{aligned}
$$

$m)$ Si indichi con $U(v)$ il massimo di $\sum_{s=1}^{m}\left|U_{s}(u, v)\right|$ sul segmento della retta

${ }^{(28)}$ Tenuto conto delle (46) e (49) risulta

$$
\begin{aligned}
\left|a_{i j}\left(\lambda, \gamma_{i}(\lambda ; u, v)\right)-a_{i j}\left(\tilde{\lambda}, \gamma_{i}(\bar{\lambda} ; u, v)\right)\right| & \leqslant \\
& \leqslant \Lambda\left\{|\lambda-\tilde{\lambda}|+\left|\gamma_{i}(\lambda ; u, v)-\gamma_{i}(\bar{\lambda} ; u, v)\right|\right\} \leqslant \Lambda\left(1+M^{[1]}\right)|\lambda-\bar{\lambda}|,
\end{aligned}
$$

e quindi

$$
\left|\frac{d a_{i j}\left(\lambda, y_{i}(\lambda ; u, v)\right)}{d \lambda}\right| \leqslant \Lambda\left(1+M^{(1)}\right)
$$


$v=$ cost. appartenente al campo $\delta$; allora dalle $(60)$ si ottengono le

$$
\begin{aligned}
\left|U_{j}(0, v)\right| \leqslant & H_{2}\left\{\nu_{2} L_{2}\left|\int_{0}^{v} \sum_{s=1}^{m}\right| U_{s}(0, \mu)|d \mu|+\right. \\
& \left.+\left(L_{2}+\Lambda M^{[11}\right) \sum_{i=v_{1}+v_{2}+1}^{m} \mid \int_{\varphi_{i}(0, v)}^{0} U\left(\gamma_{i}(\lambda ; 0, v)\right) d \lambda\right\}, \quad(j=1, \ldots, m) .
\end{aligned}
$$

Posto $\mu=\gamma_{i}(\lambda ; 0, v)$ e tenuto conto che $\gamma_{i}\left(\varphi_{i}(0, v) ; 0, v\right)=0, \gamma_{i}(0 ; 0, v)=v$ e che inoltre (cfr. le (46), (49))

$$
\frac{d \mu}{d \lambda}=R_{i}(\lambda, \mu), \quad\left|\frac{d \mu}{d \lambda}\right| \geqslant \frac{1}{k},
$$

risulta

$$
\mid \int_{\varphi_{i}(0, v)}^{0} U\left(\gamma_{i}(\lambda ; 0, v) d \lambda|=| \int_{0}^{v} U(\mu) \frac{d \lambda}{d \mu} d \mu|\leqslant k| \int_{0}^{v} U(\mu) d \mu \mid,\right.
$$

e sostituendo nelle (61)

$$
\begin{aligned}
\left|U_{j}(0, v)\right| \leqslant H_{2}\left\{v_{2} L_{2} \mid \int_{0}^{v}\right. & \sum_{s=1}^{m}\left|U_{s}(0, \mu)\right| d \mu \mid+ \\
& \left.+\left(L_{2}+\Lambda M^{[1]}\right)\left(m-\nu_{1}-\nu_{2}\right) k\left|\int_{0}^{v} U(\mu) d \mu\right|\right\}, \quad(j=1, \ldots, m) .
\end{aligned}
$$

Sommando per $j=1, \ldots, m$ e applicando il Lemma di Gronwall si ottiene successivamente

$$
\left\{\begin{aligned}
\sum_{j=1}^{m}\left|U_{j}(0, v)\right| \leqslant m H_{2}\left\{v_{2} L_{2}\left|\int_{0}^{y} \sum_{j=1}^{m}\right| U_{j}(0, \mu)|d \mu|\right. & + \\
& \left.+\left(L_{2}+\Lambda M^{[\mu]}\right)\left(m-v_{1}-v_{2}\right) k\left|\int_{0}^{v} U(\mu) d \mu\right|\right\} \\
\sum_{j=1}^{m}\left|U_{j}(0, v)\right| \leqslant m H_{2}\left(m-v_{1}-v_{2}\right)\left(L_{2}\right. & \left.+\Lambda M^{[1]}\right) k\left|\int_{0}^{v} \exp \left[m H_{\dot{2}} v_{2} L_{2}|v-\mu|\right] U(\mu) d \mu\right|
\end{aligned}\right.
$$

Dalle (59), tenuto conto delle (62) e del modo, nel quale è stata definita la fun- 
zione $U(v)$, si ottengono le

$$
\begin{aligned}
& \left|U_{j}(u, v)\right| \leqslant H_{1}\left\{m H H_{2} v_{1}\left(m-v_{1}-v_{2}\right)\left(L_{2}+A M^{[1]}\right) k \exp \left[m H_{2} L_{2} v_{2} v_{0}\right] \cdot\right. \\
& \quad \cdot\left|\int_{0}^{v} U(\mu) d \mu\right|+v_{1} L_{2}\left|\int_{0}^{u} \sum_{s=1}^{m}\right| U_{s}(\lambda, v)|d \lambda|+v_{2} L_{2}\left|\int_{0}^{v} U(\mu) d \mu\right|+ \\
& \left.+\left(L_{2}+\Lambda M^{(11)}\right) \sum_{i=\gamma_{1}+v_{2}+1}^{m}\left|\int_{\varphi_{i}(u, v)}^{u} U\left(\gamma_{i}(\lambda ; u, v)\right) d \lambda\right|\right\}, \quad(j=1, \ldots, m),
\end{aligned}
$$

dove $v_{0}$ è il massimo di $|v|$ nel campo $\delta$.

In modo analogo a quanto fatto più sopra, posto $\mu=\gamma_{i}(\lambda ; u, v)$, e tenuto conto che $\gamma_{i}\left(\varphi_{i}(u, v) ; u, v\right)=0, \gamma_{i}(u ; u, v)=v$, si ottiene subito

$$
\left|\int_{q_{i}(u, v)}^{u} U\left(\gamma_{i}(\lambda ; u, v)\right) d \lambda\right|=\left|\int_{0}^{v} U(\mu) \frac{d \lambda}{d \mu} d \mu\right| \leqslant k\left|\int_{0}^{v} U(\mu) d \mu\right|
$$

e quindi

$$
\left|U_{j}(u, v)\right| \leqslant H_{1}\left\{v_{1} L_{2}\left|\int_{0}^{u} \sum_{s=1}^{m_{m}}\right| U_{s}(\lambda, v)|d \lambda|+K\left|\int_{0}^{v} U(\mu) d \mu\right|\right\}, \quad(j=1, \ldots, m),
$$

dove $K$ è una costante, di cui è facile scrivere l'espressione esplicita $\left.{ }^{(29}\right)$.

Sommando le (63) per $j=1, \ldots, m$, si ottengono le

$$
\sum_{j=1}^{m}\left|U_{j}(u, v)\right| \leqslant m H_{1}\left\{\nu_{1} L_{2}\left|\int_{0}^{u} \sum_{j=1}^{m}\right| U_{j}(\lambda, v)|d \lambda|+K\left|\int_{0}^{v} U(\mu) d \mu\right|\right\} .
$$

In quest'ultima disuguaglianza si pensi $u$ come variabile indipendente e $v$ come un parametro; applicando il Lemma di Gronwall, e indicando con $\alpha$ il maggiore dei numeri $a_{1}, a_{2}$ risulta

$$
\sum_{j=1}^{m}\left|D_{j}(u, v)\right| \leqslant m H_{1} K \exp \left[m H_{1} v_{1} L_{2} \alpha\right]\left|\int_{0}^{v} U(\mu) d \mu\right| .
$$

In corrispondenza ad ogni valore $v$, che sia ordinata di punti del campo $\delta$, la (64) vale per ogni $u$, tale che il punto $(u, v)$ appartenga al campo $\delta$, e quindi vale, in

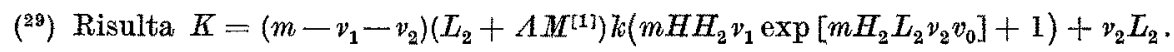


particolare, nel punto, in cui $\sum_{j=1}^{m}\left|U_{j}(u, v)\right|$ raggiunge il suo massimo $U(v)$; è dunque

$$
U(v) \leqslant m H_{1} K \exp \left[m H_{1} \nu_{1} L_{2} \alpha\right]\left|\int_{0}^{p} U(\mu) d \mu\right|
$$

da cui, come è noto, segue subito $U(v)=0$ per ogni valore di $v$, che sia ordinata di punti del campo $\delta$; quindi è identicamente in tutto $\delta$

$$
\sum_{j=1}^{m}\left|U_{j}(u, v)\right|=0 ; \quad \zeta_{j}(u, v)=\zeta_{i}^{*}(u, v), \quad(j=1, \ldots, m)
$$

vale a dire in tutto $\Delta$ è identicamente

$$
z_{j}(x, y)=z_{j}^{*}(x, y), \quad(j=1, \ldots, m)
$$

ed è quindi provato il teorema di unicità.

OsservazroNe. - Nell'enunciato del teorema di unicità le funzioni $f_{i}\left(x, y ; z_{1}, \ldots, z_{m}\right)$ sono supposte lipschitziane nel complesso delle variabili $\left(z_{1}, \ldots, z_{m}\right)$, con costante $L_{1}$ indipendente da $(x, y)$ (cf. la (13)); l'ipotesi può essere resa alquanto più ampia, nel senso che è sufficiente supporre che, comunque siano le costanti $Y_{1}^{(0)}>0, \Omega_{j}^{(0)}>0$, $(j=1, \ldots, m)$, esiste una costante $L_{1}^{(0)}$, tale che per ogni $x$ di $\left(-a_{1}, a_{2}\right)$, per ogni $y$ con $|y| \leqslant T^{(0)}$, e per tutte le coppie di $m$-ple reali $\left(z_{1}, \ldots, z_{m}\right),\left(\bar{z}_{1}, \ldots, \bar{z}_{m}\right)$; soddisfacenti le $\left|z_{j}\right| \leqslant \Omega_{\xi}^{(0)},\left|\vec{z}_{i}\right| \leqslant \Omega_{j}^{(0)},(j=1, \ldots, m)$, valgono le

$$
\left|f_{i}\left(x, y ; z_{1}, \ldots, z_{m}\right)-f_{i}\left(x, y ; \bar{z}_{1}, \ldots, \bar{z}_{m}\right)\right| \leqslant L_{1}^{(m)} \sum_{j=1}^{m}\left|z_{j}-\bar{z}_{i}\right|, \quad(i=1, \ldots, m) .
$$

\section{3. - Un caso particolare.}

Per $-a_{1} \leqslant x \leqslant a_{2}$ sia

$b_{i j}(x) \equiv 0, \quad\left(i=1, \ldots, v_{1}, j=1, \ldots, m ; i \neq j\right) ; \quad b_{i i}(x)=1, \quad\left(i=1, \ldots, v_{1}\right)$,

Le condizioni (17), (18) divengono più semplicemente

$$
\left|\begin{array}{c}
A_{v_{1}+1, v_{1}+1}\left(x, \eta_{2}(x)\right) \ldots A_{v_{1}+1, m}\left(x, \eta_{2}(x)\right) \\
\ldots \ldots \ldots \ldots \ldots \ldots \ldots \ldots \ldots \ldots \ldots \ldots \ldots \\
A_{v_{1}+1, m}\left(x, \eta_{2}(x)\right) \ldots A_{m, m}\left(x, \eta_{2}(x)\right)
\end{array}\right| \neq 0,
$$

in tutto l'intervallo $-a_{1} \leqslant x \leqslant a_{2}, \mathrm{e}$

$$
Z_{i}(0)=Q_{i}(0), \quad\left(i=1, \ldots, v_{1}\right)
$$

e la (20) diviene

$$
z_{i}\left(x, \eta_{2}(x)\right)=G_{i}(x), \quad\left(i=1, \ldots, y_{1}\right)
$$


vale a dire nei punti della caratteristica $y=\eta_{2}(x)$ sono prefissati $\mathrm{i}$ valori di $v_{1}$ tra le funzioni (1); il teorema di unicità assicura che non può esistere più di un integrale (in senso generalizzato) del sistema (I), tale che ad esso appartenga la curva caratteristica (5) e che nei punti della curva $y=\eta_{2}(x)$ soddisfi le condizioni $\left(20^{\prime}\right)$.

\section{§ 2.}

\section{4. - Teorema di dipendenza continua dai dati.}

Valgano le ipotesi del teorema di unicità (n.2); assieme alla curva caratteristica (5) del sistema corrispondente alla radice caratteristica $\sigma_{\mathbf{1}}(x, y)$, sia assegnata la curva caratteristica dello stesso sistema

$$
y=\eta_{1}(x), z_{1}=Z_{1}^{*}(x), \ldots, z_{m}=Z_{m}^{*}(x), \quad\left(-a_{1} \leqslant x \leqslant a_{2}\right),
$$

dove le funsioni $Z_{i}^{*}(x),(i=1, \ldots, m)$ sono lipschitziane in $\left(-a_{1}, a_{2}\right) ;$ siano assegnate le funzioni $G_{i}^{*}(x),\left(i=1, \ldots, v_{1}\right)$, continue in $\left(-a_{1}, a_{2}\right)$ e soddisfacenti le

$$
\sum_{j=1}^{m} b_{i j}(0) Z_{j}^{*}(0)=G_{i}^{*}(0), \quad\left(i=1, \ldots, v_{1}\right) .
$$

Nel campo 4 (definito nel n. 2 d)) siano definite le due m-ple di funsioni

$$
\begin{aligned}
& z_{1}=z(x, y), \ldots, z_{m}=z_{m}(x, y) \\
& z_{1}=z_{1}^{*}(x, y), \ldots, z_{m}=z_{m}^{*}(x, y),
\end{aligned}
$$

lipschitziane nel campo $\Delta$, e costituenti due soluzioni (in senso generalizzato) del sistema (I); assieme alle (19), (20) valgano le

$$
z_{i}^{*}\left(x, \eta_{1}(x)\right)=Z_{i}^{*}(x), \quad(i=1, \ldots, m),
$$

per $-a_{1} \leqslant x \leqslant a_{2}, e$

$$
\sum_{j=1}^{m} b_{i j}(x) z_{i}^{*}\left(x, \eta_{2}(x)\right)=G_{i}^{*}(x), \quad\left(i=1, \ldots, v_{1}\right),
$$

nei punti della curva (14) appartenenti al campo 4 . Allora, dato un numero \& positivo arbitrario, si può determinare un numero positivo $\sigma$ in modo che, per

$$
\left|Z_{i}(x)-Z_{i}^{*}(x)\right|<\sigma,(i=1, \ldots, m) ; \quad\left|Q_{i}(x)-G_{i}^{*}(x)\right|<\sigma,\left(i=1, \ldots, v_{1}\right)
$$

in tutto $\left(-a_{1}, a_{2}\right)$, risulti

$$
\sum_{j=1}^{m}\left|z_{j}(x, y)-z_{j}^{*}(x, y)\right|<\varepsilon
$$

in tutto il campo 4. 
a) Mediante il cambiamento di variabili stabilito dalle (35), (35 $)$ (cfr. n. $2 f)$ ), si definiscono nel campo $\delta$ del piano $(u, v)$ le funzioni

$$
\begin{array}{ll}
\zeta_{i}(u, v)=z_{i}(\xi(u, v), \eta(u, v)), & (i=1, \ldots, m), \\
\zeta_{i}^{*}(u, v)=z_{i}^{*}(\xi(u, v), \eta(u, v)), & (i=1, \ldots, m) .
\end{array}
$$

Le funzioni $\zeta_{i}(u, v)$ soddisfano le relazioni (52), (53) (cfr. n. $2 j$ ), $\left.k\right)$ ), e le $\zeta_{i}^{*}(u, v)$ relazioni del tutto analoghe. Posto

$$
U_{i}(u, v)=\zeta_{i}(u, v)-\zeta_{i}^{*}(u, v), \quad(i=1, \ldots, m)
$$

e inoltre

$$
\begin{array}{ll}
\Phi_{i}(u)=Z_{i}(u)-Z_{i}^{*}(u), & (i=1, \ldots, m), \\
\Psi_{i}(v)=G_{i}(v)-G_{i}^{*}(v), & \left(i=1, \ldots, v_{1}\right),
\end{array}
$$

sottraendo dalle (52), (53) le relazioni analoghe relative alle funzioni $\left(38^{*}\right)$ si trovano, nel caso attuale, le

$$
\begin{aligned}
& U_{j}(u, v)=\sum_{i=1}^{v_{i j}} \alpha_{i j}(u, v)\left\{\sum_{s=1}^{m} a_{i s}(0, v) U_{s}(0, v)+\right. \\
& +\sum_{s=1}^{m} \int_{0}^{n} \frac{\partial a_{i s}(\lambda, v)}{\partial \lambda} U_{s}(\lambda, v) d \lambda+\int_{0}^{u}\left[F_{i}\left(\lambda, v ; \zeta_{1}(\lambda, v), \ldots, \zeta_{m}(\lambda, v)\right)-\right. \\
& \left.\left.-F_{i}\left(\lambda, v ; \zeta_{1}^{*}(\lambda, v), \ldots, \zeta_{m}^{*}(\lambda, v)\right)\right] d \lambda\right\}+\sum_{i=v_{1}+1}^{\eta_{1}+v_{z}} \alpha_{i j}(u, v)\left\{\sum_{s=1}^{m} a_{i s}(u, 0) \Phi_{s}(u)+\right. \\
& +\sum_{s=1}^{m} \int_{0}^{v} \frac{\partial a_{i s}(u, \mu)}{\partial \mu} U_{s}(u, \mu) d \mu+ \\
& \left.+\int_{0}^{v}\left[F_{i}\left(u, \mu ; \zeta_{1}(u, \mu), \ldots, \zeta_{m}(u, \mu)\right)-F_{i}\left(u, \mu ; \zeta_{1}^{*}(u, \mu), \ldots, \zeta_{m}^{*}(u, \mu)\right)\right] d \mu\right\}+ \\
& +\sum_{i=v_{1}+v_{\mathrm{g}}+1}^{m} \alpha_{i j}(u, v)\left\{\sum_{s=1}^{m} a_{i s}\left(\varphi_{i}(u, v), 0\right) \Phi_{s}\left(\varphi_{i}(u, v)\right)+\right. \\
& +\sum_{s=1}^{m_{m}} \int_{Q_{i}(u, v)}^{u} \frac{d a_{i s}\left(\lambda, \gamma_{i}(\lambda ; u, v)\right)}{d \lambda} U_{s}\left(\lambda, \gamma_{i}(\lambda ; u, v)\right) d \lambda+ \\
& +\int_{\varphi_{i}(u, v)}^{u}\left[F_{i}\left(\lambda, \gamma_{i}(\lambda ; u, v) ; \zeta_{1}\left(\lambda, \gamma_{i}(\lambda ; u, v)\right), \ldots, \zeta_{m}\left(\lambda, \gamma_{i}(\lambda ; u, v)\right)\right)-\right.
\end{aligned}
$$

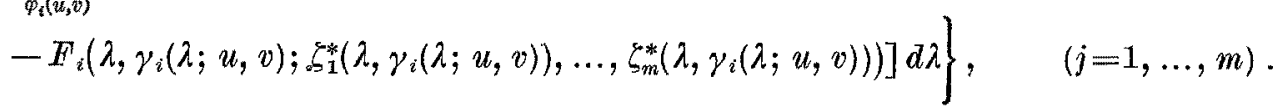




$$
U_{j}(0, v)=\sum_{i=1}^{v_{1}} \beta_{i j}(v) \Psi_{i}(v)+\sum_{i=\psi_{1}+1}^{v_{1}+p_{s}} \beta_{i j}(v)\left\{\sum_{s=1}^{m} a_{i s}(0,0) \Phi_{s}(0)+\right.
$$

$+\sum_{s=1}^{m} \int_{0}^{v} \frac{\partial a_{i s}(0, \mu)}{\partial \mu} U_{s}(0, \mu) d \mu+\int_{0}^{v}\left[F_{i}\left(0, \mu ; \zeta_{1}(0, \mu), \ldots, \zeta_{m}(0, \mu)\right)-\right.$

$\left.\left.-F_{i}\left(0, \mu ; \zeta_{1}^{*}(0, \mu), \ldots, \zeta_{m}^{*}(0, \mu)\right)\right] d \mu\right\}+\sum_{i=y_{1} \neq v_{2}+1}^{m_{m}} \beta_{i j}(v)\left\{\sum_{s=1}^{m} a_{i s}\left(\varphi_{i}(0, v), 0\right) \Phi_{s}\left(\varphi_{i}(0, v)\right)+\right.$

$+\sum_{s=1}^{m} \int_{\phi_{i}(0, z)}^{0} \frac{d a_{i s}\left(\lambda, \gamma_{i}(\lambda ; 0, v)\right)}{d \lambda} U_{s}\left(\lambda, \gamma_{i}(\lambda ; 0, v)\right) d \lambda+$

$+\int_{\varphi_{i}(0, v)}^{0}\left[F_{i}\left(\lambda, \gamma_{i}(\lambda ; 0, v) ; \zeta_{\mathbf{1}}\left(\lambda, \gamma_{i}(\lambda ; 0, v)\right), \ldots, \zeta_{m}\left(\lambda, \gamma_{i}(\lambda ; 0, v)\right)\right)-\right.$

$\left.\left.-F_{i}\left(\lambda, \gamma_{i}(\lambda ; v) ; \zeta_{i}^{*}\left(\lambda, \gamma_{i}(\lambda ; 0, v)\right), \ldots, \zeta_{m}^{*}\left(\lambda, \gamma_{i}(\lambda ; 0, v)\right)\right)\right] d \lambda\right\}, \quad(j=1, \ldots, m)$.

b) Dalle (69), (70), ragionando come nel n. 2l), seguono subito le

$$
\begin{aligned}
& \left|U_{3}(u, v)\right| \leqslant H_{\mathbf{1}}\left\{\gamma_{1} H \sum_{s=1}^{m}\left|U_{s}(0, v)\right|+\right. \\
& +v_{1} L_{2}\left|\int_{0}^{u} \sum_{s=1}^{m}\right| U_{s}(\lambda, v)|d \lambda|+m\left(m-v_{1}\right) H \sigma+v_{2} L_{2}\left|\int_{0}^{v} \sum_{s=1}^{m}\right| U_{s}(u, \mu)|d \mu|+ \\
& \left.+\left(L_{2}+\Lambda M^{[1]}\right) \sum_{i=y_{1}+y_{2}+1}^{m}\left|\int_{p_{i}(u, v v)}^{m_{s}} \sum_{s=1}^{n}\right| U_{s}\left(\lambda, \gamma_{i}(\lambda ; u, v)\right)|d \lambda|\right\}, \quad(j=1, \ldots, m) . \\
& \left|U_{j}(0, v)\right| \leqslant H_{2}\left\{v_{1} \sigma+m\left(m-v_{1}\right) H \sigma+v_{2} L_{2}\left|\int_{0}^{v} \sum_{s=1}^{m}\right| U_{s}(0, \mu)|d \mu|+\right. \\
& \left.+\left(L_{\mathrm{s}}+\Lambda M^{[1]}\right) \sum_{i=v_{1}+v_{3}+1}^{m_{2}}\left|\int_{\phi_{i}(0, v)}^{0} \sum_{s=1}^{m}\right| U_{s}\left(\lambda, \gamma_{i}(\lambda ; 0, v)\right)|d \lambda|\right\}, \quad(j=1, \ldots, m) \text {. }
\end{aligned}
$$

Indicato, come nel n. $2 m)$, con $U(v)$ il massimo di $\sum_{s=1}^{m}\left|U_{s}(u, v)\right|$ nel segmento della retta $v=$ cost. appartenente al campo $\delta$, dalle (72) si ottengono le

$$
\begin{aligned}
\left|U_{j}(0, v)\right| \leqslant H_{2}\left\{\nu_{1} \sigma+m\left(m-v_{1}\right) H \sigma+v_{2} L_{2}\left|\int_{0}^{v} \sum_{s=1}^{m}\right| U_{s}(0, \mu)|d \mu|+\right. \\
+\left(L_{2}+A M^{[11)} \sum_{i=\nu_{1}+\nu_{2}+1}^{n_{2}}\left|\int_{\phi_{i}(0, v)}^{0} U\left(\gamma_{i}(\lambda ; 0, v)\right) d \lambda\right|\right\}, \quad(j=1, \ldots, m) .
\end{aligned}
$$

14 - Annali di Matematica 
Ragionando come nel n. $2 m)$, posto $\mu=\gamma_{i}(\lambda ; 0, v)$, si trova

$$
\mid \int_{\phi_{i}(0, v)}^{0} U\left(\gamma_{i}(\lambda, 0, v) d \lambda|\leqslant k| \int_{0}^{v} U(\mu) d \mu \mid\right.
$$

sostituendo nelle (73), e poi sommando per $j=1, \ldots, m$, risulta

$$
\begin{aligned}
\sum_{j=1}^{m}\left|U_{j}(0, v)\right| \leqslant m H_{2}\left\{v_{1} \sigma+m\left(m-v_{1}\right) H \sigma+v_{2} L_{2}\left|\int_{0}^{v} \sum_{j=1}^{m}\right| U_{j}(0, \mu)|d \mu|+\right. & \\
& \left.+\left(m-v_{1}-v_{2}\right)\left(L_{2}+\Lambda M^{[1]}\right) k\left|\int_{0}^{v} U(\mu) d \mu\right|\right\}
\end{aligned}
$$

da cui per il Lemma di Gronwall

$$
\begin{aligned}
\sum_{j=1}^{m}\left|U_{j}(0, v)\right| \leqslant m H_{2}\left\{\left(v_{1}\right.\right. & \left.+m\left(m-v_{1}\right) H\right) \sigma \exp \left[m H_{2} \nu_{2} L_{2}|v|\right]+ \\
& \left.+\left(m-v_{1}-v_{2}\right)\left(L_{2}+\Lambda M^{[11}\right) k\left|\int_{0}^{v} \exp \left[m H_{2} v_{2} L_{2}|v-\mu|\right] U(\mu) d \mu\right|\right\} .
\end{aligned}
$$

In virtù di quest'ultima relazione, tenendo conto del modo, nel quale è stata introdotta la funzione $U(v)$, dalle (71) si ottengono le

$$
\left|U_{j}(u, v)\right| \leqslant H_{1}\left\{B \sigma+m v_{1}\left(m-v_{1}-v_{2}\right) H H_{2}\left(L_{2}+A M^{[1]}\right) k\right.
$$

$\cdot\left|\int_{0}^{v} \exp \left[m H_{2} v_{2} L_{2}|v-\mu|\right] U(\mu) d \mu\right|+v_{1} L_{2}\left|\int_{0}^{u} \sum_{\varepsilon=1}^{m}\right| U_{j}(\lambda, v)|d \lambda|+$

$\left.+v_{2} L_{2}\left|\int_{0}^{v} U(\mu) d \mu\right|+\left(L_{2}+\Lambda M^{[1]}\right) \sum_{i=v_{1}+v_{2}+1}^{m_{n}}\left|\int_{q_{i}(u, v)}^{u} U\left(\gamma_{i}(\lambda ; u, v)\right) d \lambda\right|\right\}, \quad(j=1, \ldots, m)$,

dove

$$
B=m v_{1} H H_{2}\left(v_{1}+m\left(m-v_{1}\right) H\right) \exp \left[m H_{2} v_{2} L_{2} v_{0}\right]+m H\left(m-v_{1}\right),
$$

e $v_{0}$ indica il massimo di $|v|$ nel campo $\delta$.

Come nel n. $2 m)$, posto $\mu=\gamma_{i}(\lambda ; u, v)$, si trova

$$
\left|\int_{\varphi_{i}(u, v)}^{u} U\left(\gamma_{i}(\lambda ; u, v)\right) d \lambda\right| \leqslant k\left|\int_{0}^{v} U(\mu) d \mu\right|, \quad\left(i=\nu_{1}+\nu_{2}+1, \ldots, m\right) .
$$


Sostituendo nelle (74), e sommando queste ultime per $j=1, \ldots, m$, risulta

$$
\sum_{j=1}^{m}\left|U_{j}(u, v)\right| \leqslant m H_{1}\left\{B \sigma+\nu_{1} L_{2}\left|\int_{0}^{u} \sum_{j=1}^{m}\right| U_{j}(\lambda, v)|d \lambda|+K\left|\int_{0}^{v} U(\mu) d \mu\right|\right\}
$$

dove $K$ è la costante introdotta nel n. $2 m$ ). Dalla (75), in cui si intenda $u$ come variabile indipendente e $v$ come parametro, si ottiene, per il Lemma di Gronwall

$$
\sum_{j=1}^{m}\left|U_{j}(u, v)\right| \leqslant m H_{1}\left\{B \sigma+K\left|\int_{0}^{v} U(\mu) d \mu\right|\right\} \exp \left[m H_{1} \nu_{1} L_{2} \alpha\right]
$$

dove $\alpha$ è il maggiore dei due numeri $a_{1}, a_{2}$; da questa segue immediatamente (cfr. n. $2 m)$ )

$$
U(v) \leqslant m H_{1}\left\{B \sigma+K\left|\int_{0}^{v} \delta(\mu) d \mu\right|\right\} \exp \left[m H_{1} \nu_{1} L_{2} \alpha\right]
$$

da cui, applicando ancora il Lemma di Gronwall e ponendo $C=\exp \left[m H_{1} \nu_{1} L_{2} \alpha\right]$, si ottiene

$$
U(v) \leqslant m H_{1} B C \exp \left[m H_{1} K C v_{0}\right] \sigma .
$$

Allora per ogni $v$, che sia ordinata di punti del campo $\delta$, risulta

$$
U(v)<\varepsilon, \text { se ̀̀ } \sigma<\frac{\varepsilon}{m H_{1} C B} \exp \left[-m H_{1} K C v_{0}\right] .
$$

Tenuto conto delle (54) e del modo, nel quale è stata definita la funzione $U(v)$, in tutto $\delta$ è anche

$$
\sum_{j=1}^{m}\left|\zeta_{j}(u, v)-\zeta_{i}^{*}(u, v)\right|<\varepsilon,
$$

e quindi, in virtù delle (38), (38*), e del cambiamento di variabili $(35),\left(35_{1}\right)$, risulta in tutto $\triangle$

$$
\sum_{j=1}^{m}\left|z_{i}(x, y)-z_{i}^{*}(x, y)\right|<\varepsilon,
$$

se ̀̀

$$
\sigma<\frac{\varepsilon}{m H_{1} \sigma B} \exp \left[-m H_{1} K C v_{0}\right]
$$




\section{5. - Complementi.}

a) Vale una osservazione del tutto analoga a quella fatta alla fine del n. 2.

b) Anche per il teorema di dipendenza continua dai dati si può enunciare facilmente il risultato particolare, contenuto nel n. 3. La (18*) diviene, in questo caso, $Z_{i}^{*}(0)=G_{i}^{*}(0),(i=1, \ldots, m)$ e la $\left(20^{*}\right)$

$$
z_{i}\left(x, \eta_{2}(x)\right)=G_{i}^{*}(x), \quad\left(i=1, \ldots, v_{1}\right)
$$

\section{BIBLIOGRAFIA}

CaRathéodory C.:

[1] Vorlesungen über reelle Funktionen, Teubner, Leipzig, 1918.

Cinquini Cibrario m. - Cinquini S.:

[1] Equazioni a derivate parziali di tipo iperbolico, Monografie Matematicho del Consiglio Nazionale delle Ricerche, n. 12, Ed. Cremonese, Roma, 1964.

\section{Cinquini Cibrarto M.:}

[1] Sui tearemi di esistenza e di unicità relativi ad alcune equazioni differenziali a derivate parziali, Nota I, Rend, R. Accad. dei Lincei (VI), 13 (1931), pp. 26-31.

[2] Sui teoremi di esistenza e di unicità relativi ad alcune equazioni differenziali a derivate parziali, Nota II, Rend. R. Acead. dei Lincei (VI), 13 (1931), pp. 115-118.

[3] Sistemi di equazioni a derivate parziali in più variabili indipendenti, Seminari dell' Istituto Nazionale di Alta Matematica, 1962-63, pp. 101-122.

[4] Teoremi di esistenza per sistemi semilineari di equazioni a derivate parziali in più variabili indipendenti, Annali di Matematica (IV), 68 (1965), pp. 119-160.

[5] Teoremi di esistenza per sistemi di equazioni quasi lineari a derivate parziali in piu variabili indipendenti, Annali di Matematica (IV), 75 (1967), pp. 1-46.

[6] Proprietà delle soluzioni di sistemi di equazioni a derivate parziali, Atti dell'VIII Congresso dell'Unione Matematica Italiana, Trieste 1967, pp. 282-283.

[7] Ulteriori risultati per $i$ sistemi semilineari di equazioni a derivate parziali in pì̀ variabili indipendenti, Rend. Istituto Lombardo, 102 (1968), pp. 801-837.

[8] Olteriori risultati per sistemi di equazioni quasi lineari a derivate parziali in pì̀ variabili indipendenti, Rend. Istituto Lombardo, 103 (1969), pp. 373-407.

[9] Teoremi di esistenza per sistemi di equazioni non lineari a derivate parziali in più variabili indipendenti, Rend. Istituto Lombardo, 104 (1970), pp. 795-829.

\section{CINQUini S.:}

[1] Teoremi di unicità per sistemi di equazioni a derivate parziali, Seminario dell'Istituto Nazionale di Alta Matematica, 1962-63, pp. 206-215.

[2] Sopra un teorema di unicitd per un sistema non lineare di equazioni a derivate parziali del secondo ordine, Atti del VII Congresso dell'Unione Matematica Italiana, Genova 1963. 
[3] Sopra l'unicità della soluzione del problema di Cauohy per sistemi di equazioni a derivate parziali quasi-lineari, Atti del Convegno su le equazioni alle derivate parziali, Bologna 1967, pp. 52-57.

[4] A proposito dei sistemi di equazioni a derivate parziali di tipo iperbolico, Atti del Convegno su le equazioni alle derivate parziali, Bologna 1967, pp. 58-62.

[5] Un teorema di unicitò per sistemi di equazioni a derivate parziali quasi-lineari, Annali di Matematica (IV), 75 (1967), pp. 231-260.

[6] Sopra $i$ sistemi di equazioni a derivate parziali ai tipo iperbolico, Atti dell'VIII Congresso dell'Unione Matematica Italiana, Trieste 1967, pp. 280-281.

[7] Sopra l'iperbolicitò dei sistemi di equazioni a derivate parziali in più variabili indipendenti, Rend. Accad. dei Lincei (VIII), Nota I, 43 (1967), pp. 288-292; Nota II, 43 (1967), pp. 464-468; Nota III, 44 (1968), pp. 9-14.

[8] A proposito dei sistemi di equazioni a derivate parziali di tipo iperbalico in più variabili indipendenti, Periodico di Matematica (IV), 46 (1968), pp. 100-106.

Mo SHANe E. G.:

[1] Integration, Princeton University Press, 1967.

RADEMACHER H.:

[1] Über partielle und totale Differenzierbarkeit von Funktionen mehrever Variabeln und über Transformation der Doppelintegrale, Math. Annalen, I, 79 (1919), pp. 340-359; II, 81 (1920), pp. 52-63.

Scorza Dragont G.:

[1] Un'osservazione sulla derivata di una funsione composta, Rend. Seminario Matematico della Università di Padova, 20 (1951), pp. 462-467.

VOLPATO M.:

[1] Sulla derivabilità, rispetto a valori iniziali ed a parametri, delle soluzioni dei sistemi di equazioni differenziali ordinarie del primo ordine, Rend. Seminario Matematico della Università di Padova, 28 (1958), pp. 71-106. 